\title{
Lunar COTS: An Economical and Sustainable Approach to Reaching Mars
}

\author{
Dr. Allison F. Zunigai, Dr. Daniel Rasky ${ }^{\mathrm{ii}}$ and Robert B. Pittman ${ }^{\mathrm{iii}}$ \\ NASA Ames Research Center, Moffett Field, CA 94035 \\ Edgar Zapata ${ }^{\text {iv }}$ \\ NASA Kennedy Space Center, FL 32899 \\ and \\ Roger Lepsch ${ }^{\mathrm{v}}$ \\ NASA Langley Research Center, Hampton, VA, 23681
}

\begin{abstract}
The NASA COTS (Commercial Orbital Transportation Services) Program was a very successful program that developed and demonstrated cost-effective development and acquisition of commercial cargo transportation services to the International Space Station (ISS). The COTS acquisition strategy utilized a newer model than normally accepted in traditional procurement practices. This new model used Space Act Agreements where NASA entered into partnerships with industry to jointly share cost, development and operational risks to demonstrate new capabilities for mutual benefit. This model proved to be very beneficial to both NASA and its industry partners as NASA saved significantly in development and operational costs while industry partners successfully expanded their market share of the global launch transportation business. The authors, who contributed to the development of the COTS model, would like to extend this model to a lunar commercial services program that will push development of technologies and capabilities that will serve a Mars architecture and lead to an economical and sustainable pathway to transporting humans to Mars. Over the past few decades, several architectures for the Moon and Mars have been proposed and studied but ultimately halted or not even started due to the projected costs significantly exceeding NASA's budgets. Therefore a new strategy is needed that will fit within NASA's projected budgets and takes advantage of the US commercial industry along with its creative and entrepreneurial attributes. The authors propose a new COTS-like program to enter into partnerships with industry to demonstrate cost-effective, cis-lunar commercial services, such as lunar transportation, lunar ISRU operations, and cislunar propellant depots that can enable an economical and sustainable Mars architecture. Similar to the original COTS program, the goals of the proposed program, being notionally referred to as Lunar Commercial Orbital Transfer Services (LCOTS) program will be to: 1) reduce development and operational costs by sharing costs with industry; 2) create new markets in cis-lunar space to further reduce operational costs; and 3) enable NASA to develop an affordable and economical exploration Mars architecture. The paper will describe a plan for a proposed LCOTS program, its potential impact to an eventual Mars architecture and its many benefits to NASA, commercial space industry and the US economy.
\end{abstract}

i Project Development Manager, Ames Space Portal Office and AIAA Associate Fellow.

ii Senior Scientist, Ames Space Portal Office, and AIAA Associate Fellow.

iii Senior Engineer, Lockheed, Ames Space Portal Office, and AIAA Associate Fellow.

${ }^{\text {iv }}$ Senior Analyst, Modeling \& Analysis, and AIAA Senior Member.

${ }^{v}$ Senior Research Engineer, Systems Analysis and Concepts Directorate, and AIAA Member. 


\section{Nomenclature}

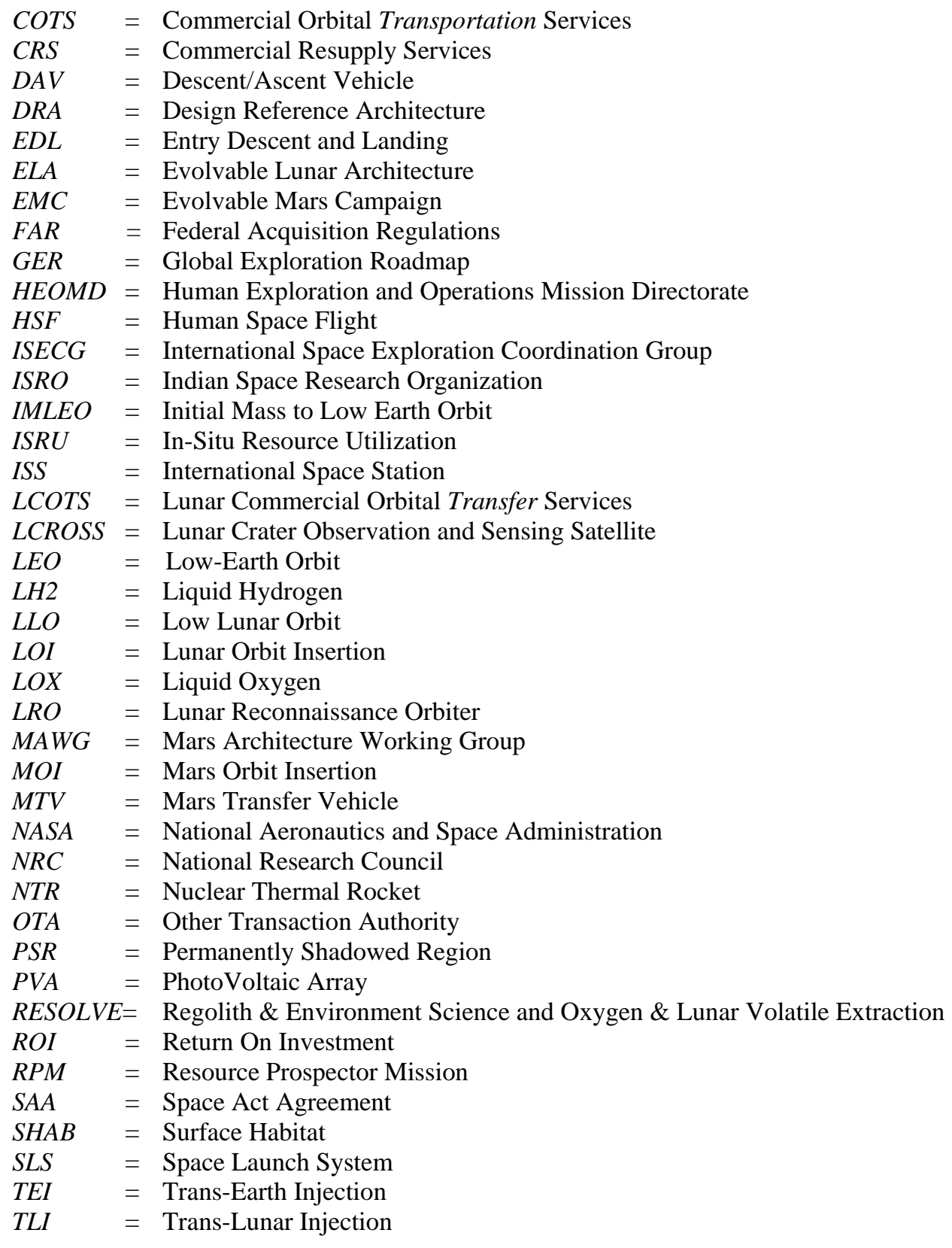

\section{Introduction}

Dresident Obama's 2010 National Space Policy ${ }^{1}$ established far-reaching exploration goals for NASA including human missions to Mars as explicitly stated in: "By the mid-2030's, send humans to orbit Mars and return them safely to Earth". This goal is further supported by the specific objectives in NASA's 2014 Strategic Plan ${ }^{2}$ which states "Expand human presence into the solar system and to the surface of Mars to advance exploration, science, innovation, benefits to humanity, and international collaboration." In response to these goals and objectives, NASA's Human Exploration and Operations Mission Directorate (HEOMD) continues to evolve plans, conduct trade studies and investigate architectures for transporting humans to Mars as described in the recent Evolvable Mars Campaign briefing ${ }^{3}$ dated April 2015.

Prior to the Evolvable Mars Campaign work, NASA's Mars Architecture Working Group (MAWG) had developed a Mars Design Reference Architecture ${ }^{4} 5.0$ (DRA5) which was released in 2009. This reference architecture described all the systems and operations needed for transporting humans to Mars. It's final two 
architecture options (summarized in Section II) estimated that approximately 800 to 1200 metric tons of Initial Mass to Low Earth Orbit (IMLEO) was needed to transfer all the necessary crew, hardware, life-support equipment and propellant to the surface of Mars and allow return for each human mission. As will be shown in Section II, most of the mass of a Mars Transfer Vehicle is propellant mass, which is on the order of 50 to $60 \%$. Therefore, to lift all this mass to LEO (where the elements would be staged and integrated), DRA5 estimated that 7 to 12 heavy-lift launch vehicles, in surges (launches very close together in time), would be needed per Mars campaign. Today, that NASA heavy-lift vehicle would be the Space Launch System (SLS).

This architecture also included major technology development challenges such as nuclear thermal rockets, aeroshell braking for Mars atmosphere, ascent/descent vehicles, ISRU plants, nuclear power stations, and a new upper stage and advanced boosters for the heavy lift vehicle, within a surge launch campaign concept. As a consequence, DRA5 resulted in very high cost estimates for its development and operational phases. Therefore, NASA's Evolvable Mars Campaign and other groups continue to conduct trade studies, investigate various technology options and opportunities for international and commercial partnerships to achieve an affordable and sustainable Mars architecture.

Concurrently, a recent 2014 report by the National Research Council (NRC) entitled "Pathways to Exploration: Rationales and Approaches for a US Program of Human Space Exploration" "also concluded that the current human spaceflight program to develop launch vehicles and spacecraft for missions beyond LEO has "unrealistic and unsustainable mission rates well below any historical precedent." The NRC estimated costs for the DRA5 architecture with a targeted first human Mars landing in mid-2030's is shown in figure 1, also from reference 5.

The NRC sand chart shows the total projected NASA Human Exploration \& Operations budget (approximately \$8B a year in 2015), both as flat and as adjusted for cost inflation, against the estimated costs for a DRA5-like Mars exploration architecture as well as estimated costs for other recommended exploration pathways such as, lunar sortie and lunar outpost. As clearly illustrated, the DRA5 architecture budgets and other exploration pathways far exceed even the most optimistic assumption of an HEOMD budget increasing on par with estimated cost inflation for this schedule-driven budget.

Furthermore, the NRC presented analysis of various approaches or pathways in its report to achieve the goal of human missions to Mars which they agree should be the ultimate goal of our national space program. They made several recommendations for implementing an affordable and sustainable program for human spaceflight beyond LEO. One of these recommendations stated:

"it was clear to the committee from its independent analysis of several pathways that a return to extended surface operations on the Moon would make substantial contributions to a strategy ultimately aimed at landing people on Mars."

They also recommended to "vigorously pursue opportunities for international and commercial collaboration in order to leverage financial resources and capabilities of other nations and commercial entities" and "continue to leverage commercial products and practices to strengthen the industrial base and increase NASA's efficiency." It is clear from these statements that the NRC finds great merit in returning to the Moon and leveraging from commercial space partnerships as much as possible to achieve an affordable and sustainable approach to reaching Mars.

A, SCHEDULE-DRIVEN ENHANCED EXPLORATION ANNUAL COST (THEN-YEAR \$)

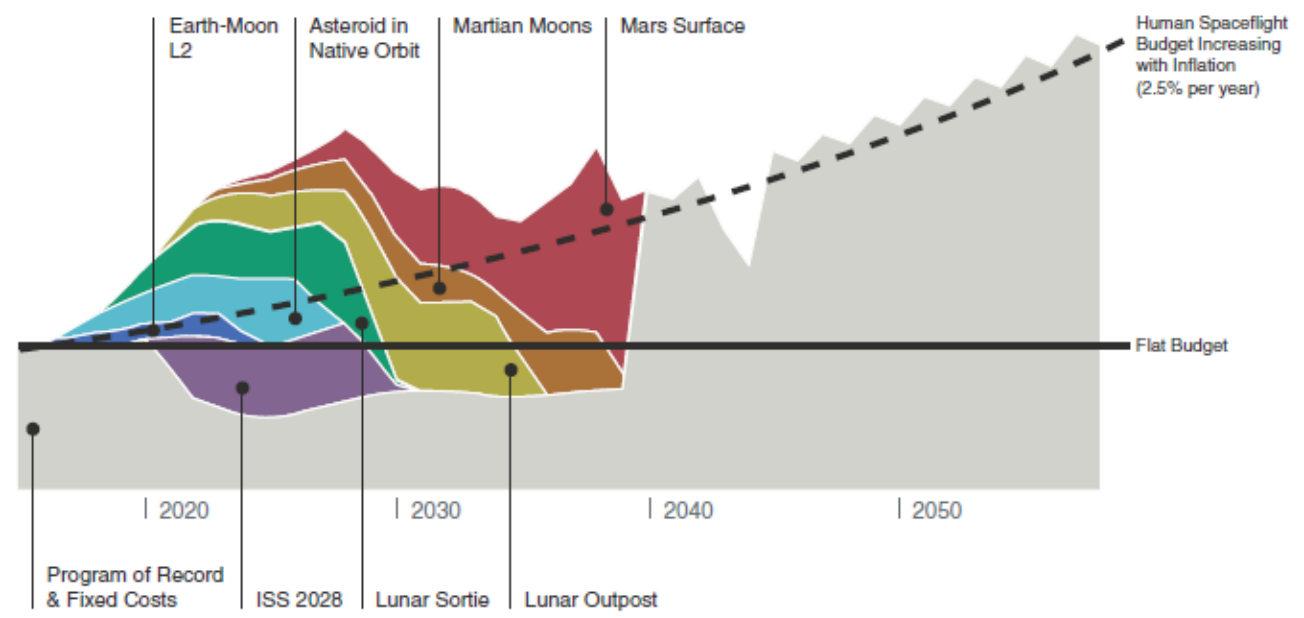

Figure 1. NASA's HSF Budget including Mars DRA5 architecture as estimated by the NRC, Ref. 5 
Additionally, the International Space Exploration Coordination Group's (ISECG) released their latest Global Exploration Roadmap ${ }^{6}$ (GER) in 2013. The ISECG represents numerous space agencies from different nations around the globe, such as, NASA, European Space Agency, Roscosmos of Russia, JAXA of Japan, ISRO of India, and the Canadian Space Agency. These participating agencies have collaborated to develop a coordinated space exploration roadmap for all the agencies to use in their domestic planning for a unified approach to exploration. The GER's long-term goal is to enable humans to explore the surface of Mars in a manner that is affordable, sustainable and productive. Towards this end, the GER also recommended that a stepwise approach be taken to reduce technical risk and make progress towards meeting exploration objectives, such as in science and technology. After examination of several stepwise approaches, the GER concluded that a return to the Moon would be most advantageous and specifically stated:

"With the goal of enabling several partners to contribute critical capabilities to future human missions, agencies note that near-term collaborative missions on the ISS, in the lunar vicinity, on the lunar surface and robotic missions may be used to simulate and better inform preparations for future international missions to Mars."

The GER also noted that ISS cargo delivery services (that resulted from COTS and Commercial Resupply Services (CRS) Programs) have demonstrated the feasibility and success of public-private partnerships to create affordable space services and products. They also noted the importance of encouraging new services and economic markets to incorporate in future planning, as stated in, "With a large number of planned lunar robotic missions in the next decade, it is considered likely that logistic services to the lunar surface will become commercially available, so they are included in the mission scenario." They also stress the importance of encouraging private sector investments by making long-term commitments as stated in, "For such private sector efforts to succeed they need the certainty of a long-term governmental commitment to space exploration." Finally, they conclude that agency leaders must focus on delivering value to the public in order to build a sustainable human space exploration endeavor that lasts decades.

Both of these reports (NRC and GER), and others ${ }^{7,8}$, have shown that in order to achieve affordability and sustainability, it is critical to embark on a stepping stone approach to demonstrate incremental successes and value to the public on our journey to Mars. It will become increasingly difficult and challenging to continue to garner support on such a costly and decades-long journey without showing tangible benefits to the public and/or the economy. These benefits may range from new consumer products, technological breakthroughs to new commercial services such as orbital tourism. A good measure of success will be substantial impacts to the economy by the addition of new markets, new products and services. Therefore it is imperative to engage and incorporate the wellestablished and emerging space industries in the planning stages of an evolvable Mars strategy to ensure a sustainable and economical approach to Mars.

A good example of spawning new commercial products and services from a NASA-sponsored program are the COTS/CRS programs. These programs were initiated to create commercial products and services at competitive prices to support the long-term, logistical cargo and crew transportation needs of ISS. To ensure competitive prices and emergence of new markets to further stimulate the economy, NASA entered into cost-sharing partnerships with industry via Space Act Agreements instead of the traditional cost-plus government contracts. By implementing this approach, or COTS model as will be explained in more detail in Section IV, NASA saved significantly in up-front development and downstream operations costs. In addition these programs resulted in the creation of new commercially available products (e.g. SpaceX's Falcon 9 launch vehicle and Dragon spacecraft and Orbital's Antares launch vehicle and Cygnus spacecraft) and services (commercial cargo delivery to ISS) that are critical and cost-saving components of the ISS program. Similarly, NASA should pursue a COTS-like acquisition approach to engage and integrate industry in its stepping-stone approach to an economical and sustainable plan to reach Mars.

As recommended by the NRC and GER reports, a necessary stepping stone approach would be a return to the Moon as it has great potential for international and commercial partnerships as well as opportunities for development of capabilities needed for future Mars missions. As shown in many scientific findings and recent reports ${ }^{9,10}$, there are an abundance of valuable resources on the Moon, (such as, $\mathrm{H} 2 \mathrm{O}, \mathrm{O} 2$ and $\mathrm{Al}$ ) that can be extracted or mined for commercial purposes (to be described more in Section III). Several studies ${ }^{11,12,13}$ have also shown that extracting these resources, such as water and oxygen, can be easily accomplished. Furthermore, the Evolvable Lunar Architecture (ELA) study, as described in reference 11, presented an affordable and economical plan for establishing a permanent commercial lunar base that can create and deliver up to $200 \mathrm{mt}$ of propellant per year to cis-lunar space for a total estimated development cost of $\$ 40 \mathrm{~B}$ over a 10-12 year period. If this plan is realized and lunar-derived propellant can be produced and delivered at economical rates, then this will have a dramatic impact to future human Mars mission concepts as the IMLEO without the propellant will be significantly reduced as well as the required number of heavy-lift launches, making future Mars concepts more affordable. 
Therefore it is recommended that a potential LCOTS program be investigated to evaluate the potential benefits to future Mars mission concepts and value to the public for sustainability. As reported in the ELA study, development of an affordable, lunar commercial industrial base to create propellant will have far-reaching benefits beyond enablement of an economical and sustainable pathway to human missions to Mars. If a lunar commercial base to support NASA's missions is realized, then this will spur the emerging space industry to create new cis-lunar markets and businesses which will result in tremendous impacts to global economy, national security, and US global leadership and the emergence of a new era of lunar colonies and human habitation on the Moon.

\section{Summary of Mars Architecture}

The Mars Design Reference Architecture (DRA 5.0) has been widely accepted as the reference architecture for transporting humans to Mars since its release in 2009. It was developed by NASA's Mars Architecture Working Group (MAWG) to fulfill NASA's need for updated and unified reference architecture for human exploration of Mars. As a result, this architecture describes in detail the systems and operations that would be used for the first three human missions to explore the surface of Mars.

Figure 2 illustrates the concept of operations for this architecture as shown in reference 4. In summary, two cargo Mars Transfer Vehicles (MTV's) are launched in components, assembled (via rendezvous and docking) in LEO and sent on a low-energy Mars trajectory from an approximate 407-km circular orbit that will take approximately 9-12 months to reach Mars orbit. These cargo MTV's will deliver the Surface Habitat (SHAB) lander system, Descent/Ascent Vehicle (DAV), aeroshells, nuclear power system, ISRU plant, several rovers and other hardware and equipment needed ahead of crew arrival. Once in Mars orbit, the DAV performs an entry, descent and landing (EDL) maneuver to land its payloads up to approximately $40 \mathrm{mt}$ to the desired landing site. Once on the surface, the surface fission reactor would be deployed to begin ISRU operations. This unit will then autonomously begin production of propellant for the ascent stage needed for human return to Mars orbit. The second cargo MTV carrying the Habitat Lander or SHAB will wait in Mars orbit until the crew MTV arrives.

Approximately 2 years after the cargo MTVs are launched, a crew of six will be launched along with its crew MTV which is also assembled in LEO. The crew MTV performs a TMI maneuver which places it on a shorter duration (approximately 6 months) transfer to Mars than the cargo MTV. Once in Mars orbit, the crew will transfer to the SHAB via the Orion crew capsule and descend to the surface. The crew will then stay on the surface for approximately 18 months performing exploration and scientific studies. They will then return to Mars orbit via the Ascent Vehicle where it will rendezvous with the crew MTV, perform a TEI maneuver and return to Earth.

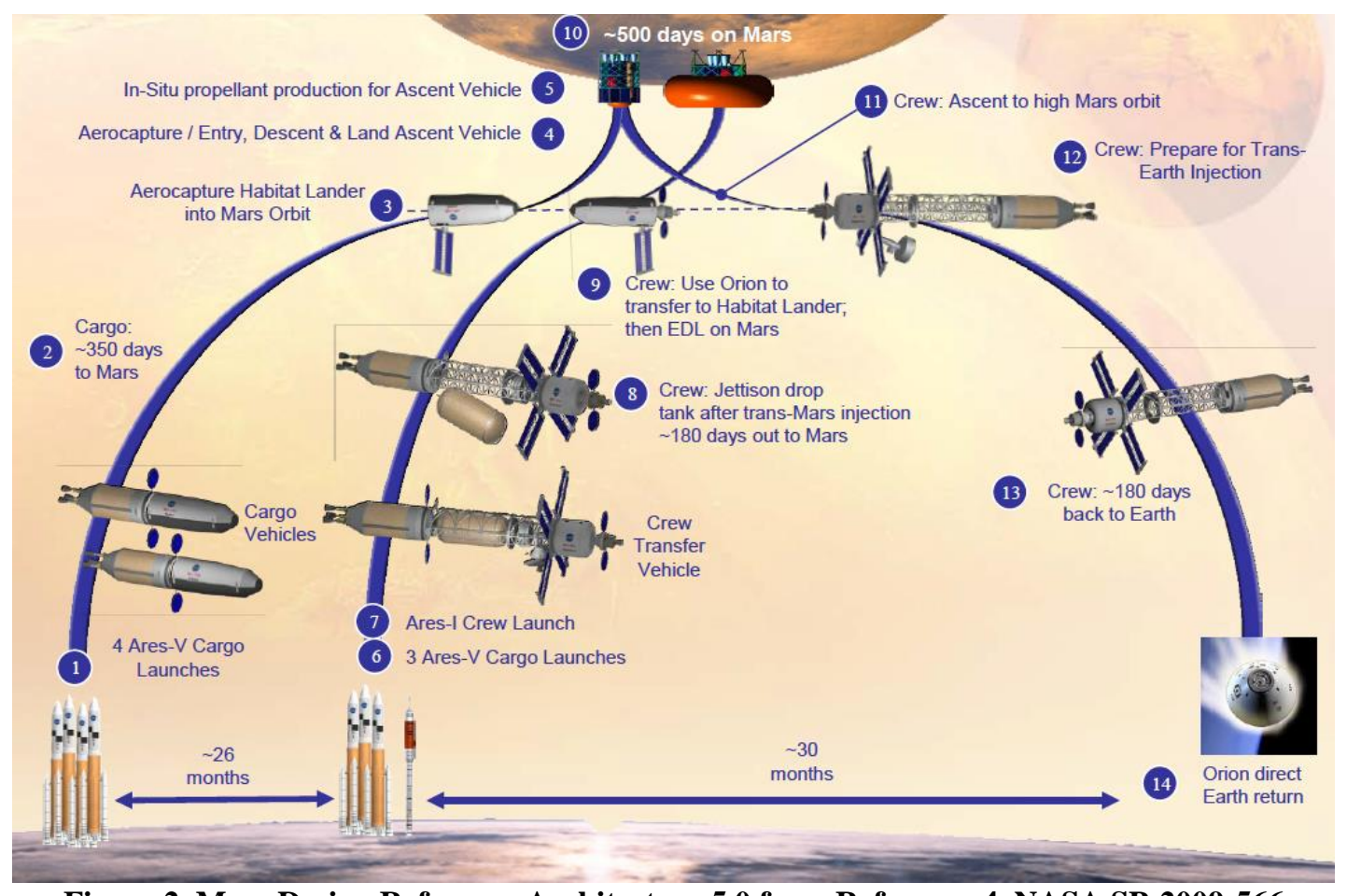

Figure 2. Mars Design Reference Architecture 5.0 from Reference 4, NASA SP-2009-566 


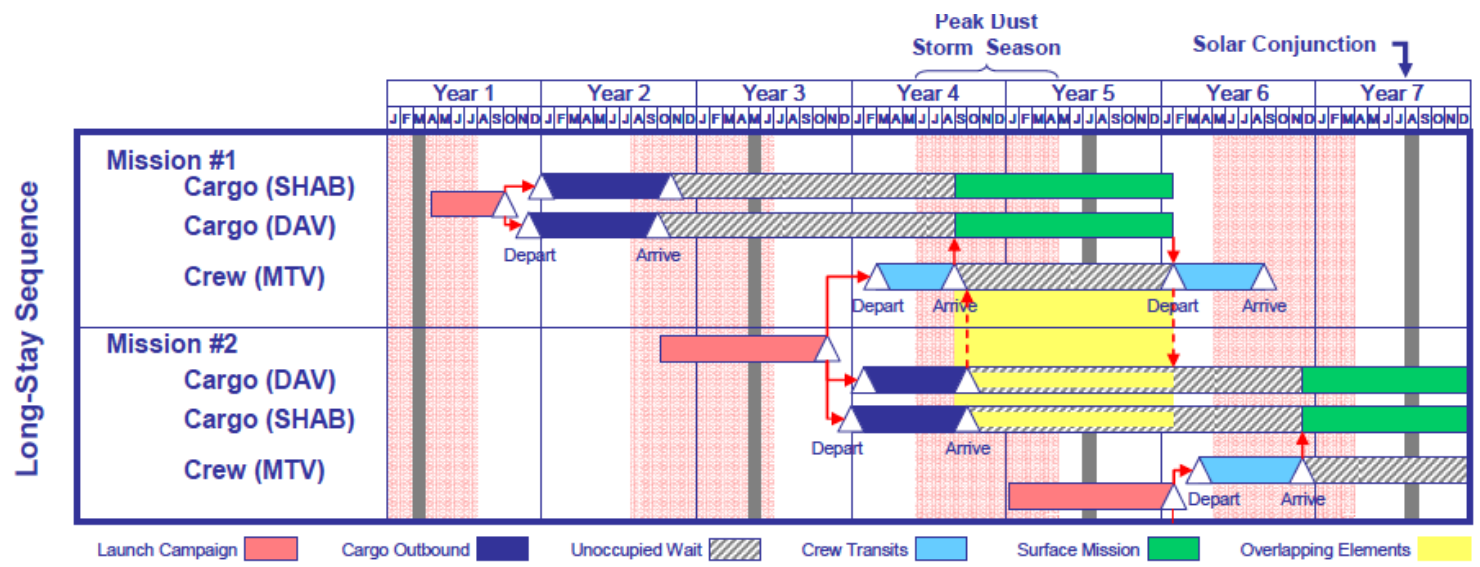

Figure 3. DRA5 Mission Sequence timelines from Reference 4, NASA SP-2009-566

The DRA5 set a minimum of three human missions for this architecture for several reasons including development time and cost to achieve the basic capability and time required to accomplish the basic program goals. These missions were planned to occur on three consecutive trajectory opportunities approximately every 2 years. Figure 3 illustrates the mission sequence timelines for two of the three missions as planned for the DRA5 architecture.

Two in-space propulsion options were presented in DRA5: a nuclear thermal rocket (NTR) stage and advanced chemical propulsion stage. The NTR common propulsion stage uses a fission-reactor-generated thermal power and consists of three 25-klbf NTR engines. The advanced chemical propulsion option was based on RL10-B2 engines supplied by LOX/LH2 propellant. The number of engines for each stage varied from 5 engines for the TMI module to 2 engines for the TEI module. Although the NTR engine was a lower TRL technology propulsion option of the two, it was selected as the preferred approach for DRA5 due to its high thrust (10's of klbf) and high specific impulse (Isp 875-950s) capability using hydrogen propellant which is twice that of today's LOX/LH2 chemical rocket engines.

Figure 4 shows a more detailed view of the crew and cargo MTVs with NTR common propulsion stages. Table 1 presents a summary of the major elements with its estimated mass listed for each. As shown in the table, the 2 cargo MTVs and $1 \mathrm{crew}$ MTV sum up to a total of approximately $800 \mathrm{mt}$ of IMLEO for each human mission to Mars.

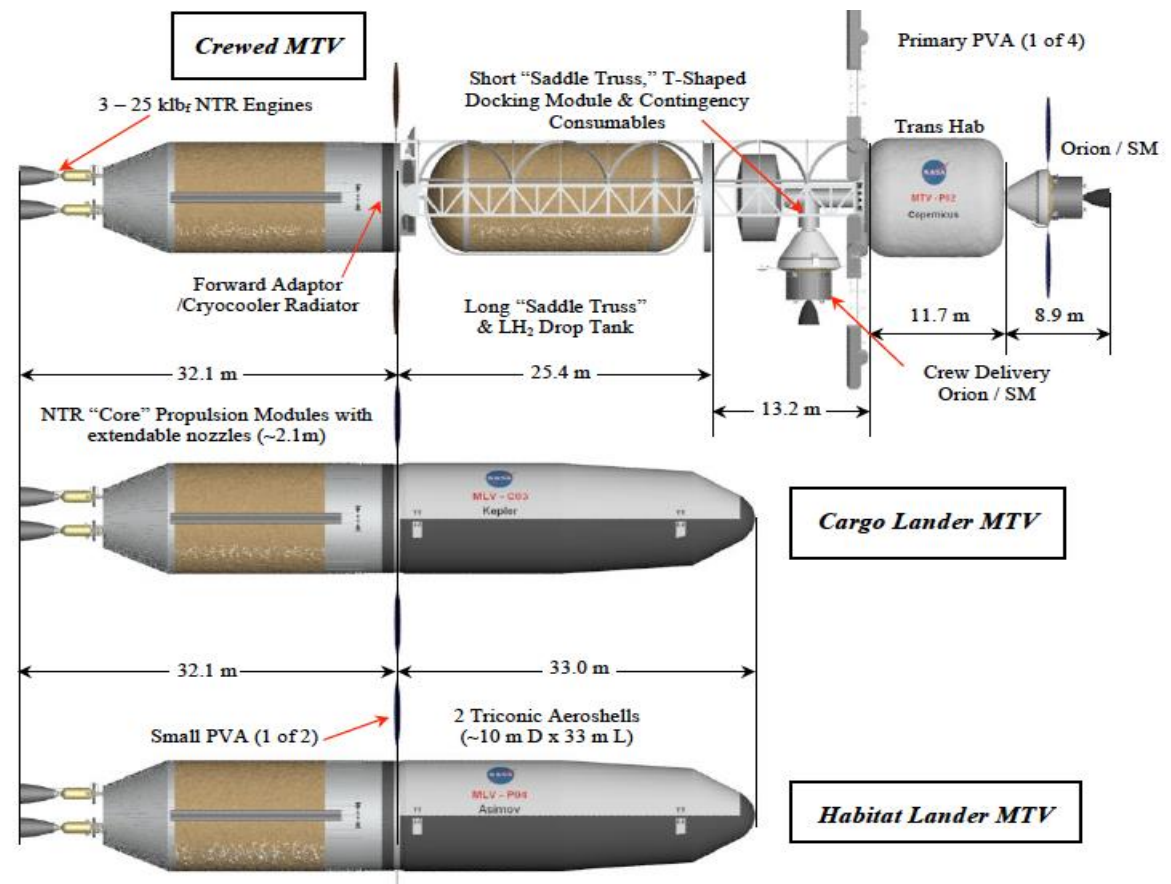

Figure 4. Crewed and Cargo MTVs based on NTR propulsion stages

6

American Institute of Aeronautics and Astronautics 
Table 1. Mass estimates (from Ref. 4) for major elements of Cargo and Crewed MTV for the NTR Option

\begin{tabular}{|c|c|c|c|}
\hline $\begin{array}{l}\text { Cargo Mars Transfer Vehicle } \\
\text { (Note all masses are in metric tons) }\end{array}$ & Mass & Crew Mars Transfer Vehicle & Mass \\
\hline $\begin{array}{l}\text { NTR common propulsion stage (includes } 87.2 \mathrm{t} \text { of } \\
\text { LH2 prop) }\end{array}$ & 133.2 & $\begin{array}{l}\text { NTR common propulsion stage (includes } 87.2 \text { of } \\
\text { LH2 prop) }\end{array}$ & 139 \\
\hline $\begin{array}{l}\text { Surface Habitat (SHAB) lander system including } \\
\text { nuclear power system, and } 2 \text { small pressurized } \\
\text { rovers }\end{array}$ & 103 & $\begin{array}{l}\text { LH2 drop tank and long saddle truss (includes } 94 \mathrm{t} \\
\text { of LH2) }\end{array}$ & 124 \\
\hline $\begin{array}{l}\text { Descent/Ascent Vehicle (DAV) system includes ISRU } \\
\text { plant, nuclear surface power plant, drill and } 2 \\
\text { unpressurized rovers }\end{array}$ & 103 & $\begin{array}{l}\text { Payload includes } 6 \text { crew, Transit Habitat, short } \\
\text { saddle truss, } 2^{\text {nd }} \text { docking module, } 2 \text { Orion/Service } \\
\text { Modules }\end{array}$ & 64 \\
\hline Total IMLEO for each Cargo MTVs includes prop & 236.2 & Total IMLEO for Crewed MTV includes prop & 327 \\
\hline Total LH2 prop for 2 cargo MTV's & 174.4 & Total LH2 prop for 1 crewed MTV & 181.2 \\
\hline Total IMLEO for 2 Cargo MTVs and 1 Crew MTV & $\sim 800$ & Total LH2 prop for 2 Cargo MTVs and 1 Crew MTV & 356 \\
\hline
\end{tabular}

Also it is worth noting from Table 1 that a total of approximately $356 \mathrm{mt}$ of LH2 propellant is needed to support all the stages of the 3 vehicles on its trajectory to Mars from LEO. The 140-metric-ton-capacity Ares V launch system concept was assumed for delivering these elements to LEO. The number of Ares V launches varied from 7 to 9 in DRA5. A report by Borowski ${ }^{14}$ concluded that a total number of 7 Ares V launches were needed for the NTRbased vehicles: 4 launches for the cargo MTVs and 3 launches for the crewed MTV.

Another report by Holladay ${ }^{15}$ analyzed several more heavy-lift options for launching all the necessary Mars elements to orbit. An interesting finding showed that only 4 Ares V launch vehicles would be necessary if the propellant was separated from the Mars elements and delivered to a LEO propellant tanker by existing commercial launch vehicles. This analysis was conducted using the advanced chemical propulsion option for the stages. Table 2 lists the estimated mass for each major element including the LOX/LH2 propellant needed. For this commercial launch option, a total of $650 \mathrm{mt}$ of propellant was estimated to be needed in orbit every 2 years to meet the cadence of missions planned for DRA5. This total showed that 54\% of the IMLEO needed for the MTV's consist of LOX/LH2 propellant. This scenario represents an excellent opportunity for a commercial market to emerge and supply the propellant needed at a more cost-effective rate than provided by heavy-lift launch vehicles. There have been several studies ${ }^{16,17}$ that have investigated commercial propellant depots in LEO as well as in cis-lunar space, as discussed previously in the ELA study, to capture this market. Section VIII below will provide an economic assessment for a cis-lunar propellant depot as well as discuss the benefits for developing such a capability.

Table 2. Mass estimates (from Ref. 4) for major elements of MTVs for the Adv. Chemical Propulsion Option

\begin{tabular}{|l|l|l|l|}
\multicolumn{1}{|c|}{$\begin{array}{c}\text { Cargo Mars Transfer Vehicle } \\
\text { (Note all masses are in metric tons) }\end{array}$} & Mass & \multicolumn{1}{c|}{ Crew Mars Transfer Vehicle } & \multicolumn{1}{|c|}{ Mass } \\
\hline $\begin{array}{l}\text { Surface Habitat (SHAB) lander system including } \\
\text { nuclear power system, and 2 pressurized rovers }\end{array}$ & 103 & $\begin{array}{l}\text { Transit Habitat Module includes 6 crew, short } \\
\text { saddle truss, 2 }{ }^{\text {nd }} \text { docking module, 2 Orion/Service } \\
\text { Modules }\end{array}$ & $\sim 100$ \\
\hline $\begin{array}{l}\text { Descent/Ascent Vehicle (DAV) lander system } \\
\text { includes ISRU plant, nuclear surface power } \\
\text { plant, drill and } 2 \text { unpressurized rovers }\end{array}$ & 103 & TEl and MOI stages & 125 \\
\hline Empty TMI Stage & 30 & 3 TMI Stages & 90 \\
\hline LOX/LH2 Propellant for each Cargo MTV & 192 & LOX/LH2 Propellant for TMI stages & $\sim 250$ \\
\hline Total IMLEO for each Cargo MTVs includes prop & $\mathbf{3 2 5}$ & Total IMLEO for Crewed MTV includes prop & $\mathbf{5 6 5}$ \\
\hline Total IMLEO for 2 Cargo MTVs and 1 Crew MTV & $\mathbf{1 2 1 5}$ & Total LOX/LH2 for 1 Mars Mission every 2 years & $\sim 650$ \\
\hline
\end{tabular}


In summary, Mars architectures to date have been characterized by:

- Large need for propellant to LEO which makes up most of the mass for a mission to Mars;

- Long development times and large budgets needed to mature technologies and demonstrate capabilities in a space environment; from habitation to landers to stages; to ISRU capabilities;

- Need for staging of mission elements in LEO, before departing to Mars, opens the possibility of commercial propellant delivery separate from the space systems elements.

\section{Lunar Resources and ISRU Capabilities}

Knowing that the Mars missions need such large amounts of propellant that may be delivered separately, all possible resources, such as the Moon, should be investigated as an option for delivery of these resources. The question naturally arises: Would it be possible to extract, process and deliver these resources from the Moon economically? To answer this question, it is important to first review and understand the resources available on the Moon followed by investigations on how to perform ISRU on the surface of the Moon economically.

There have been several remote-sensing, lunar missions in the last two decades searching for evidence of waterice in the permanently shadowed regions (PSRs) of the moon. These missions included DOD and NASA's Clementine mission launched in 1994; NASA's Lunar Prospector mission launched in 1998; ISRO's Chandrayaan-1 launched in 2008; and NASA's Lunar Reconnaissance Orbiter (LRO) launched in 2009 with mini-RF radar instrument. All these missions provided some indirect evidence that the poles may contain water ice deposits. Furthermore, LRO's mini-RF experiment results indicated that several hundred millions of tons of water may exist in the upper 2-3 $\mathrm{m}$ of the lunar surface at both poles ${ }^{18}$.

The results ${ }^{19}$ from the earlier missions (Clementine and Prospector) were debated over a decade until NASA's Lunar Crater Observation and Sensing Satellite (LCROSS) mission was launched in 2009 (together with LRO) and intentionally navigated to impact the moon to provide direct evidence of water ice. The mission's plan was to send both LCROSS and the attached upper stage, Centaur, on a trajectory to impact the Cabeus crater in the south pole region of the moon. On October 9, 2009, the Centaur crashed into that crater causing a huge debris plume as planned with LCROSS' Shepherding spacecraft following a few minutes later, collecting and relaying data from the plume back to Earth. The Shepherding spacecraft carried 9 instruments, including cameras, spectrometers and a radiometer. Results ${ }^{20}$ showed concentrations of water ice estimated at $5.6 \pm 2.9 \%$ by mass in the lunar regolith of this crater.

Further studies ${ }^{21}$ have indicated that the cumulative areas of the PSRs of the northern and southern hemisphere total an estimated area of $31,059 \mathrm{~km}^{2}$. If all this estimated area contained water ice at the estimated levels provided by the LCROSS results, then the total quantity of water contained within the uppermost meter of all the PSRs would be $2.9 \times 10^{12} \mathrm{~kg}$ (or 2900 million $\mathrm{mt}$ of water) as reported in reference 10 .

In addition to the presence of water, the LCROSS results indicated the abundances of other volatile species other than water as shown in the table below:

Table 3. Abundances of volatile species from LCROSS results (Colaprete Ref. 20)

\begin{tabular}{lcc} 
Compound & Molecules $\mathrm{cm}^{-2}$ & \% Relative to $\mathbf{H}_{2} \mathrm{O}(\mathbf{g})^{*}$ \\
\hline $\mathrm{H}_{2} \mathrm{O}$ & $5.1(1.4) \mathrm{E} 19$ & $100.00 \%$ \\
$\mathrm{H}_{2} \mathrm{~S}$ & $8.5(0.9) \mathrm{E} 18$ & $16.75 \%$ \\
$\mathrm{NH}_{3}$ & $3.1(1.5) \mathrm{E} 18$ & $6.03 \%$ \\
$\mathrm{SO}_{2}$ & $1.6(0.4) \mathrm{E} 18$ & $3.19 \%$ \\
$\mathrm{C}_{2} \mathrm{H}_{4}$ & $1.6(1.7) \mathrm{E} 18$ & $3.12 \%$ \\
$\mathrm{CO}_{2}$ & $1.1(1.0) \mathrm{E} 18$ & $2.17 \%$ \\
$\mathrm{CH}_{3} \mathrm{OH}$ & $7.8(42) \mathrm{OH} 17$ & $1.55 \%$ \\
$\mathrm{CH}_{4}$ & $3.3(3.0) \mathrm{E} 17$ & $0.65 \%$ \\
$\mathrm{OH}$ & $1.7(0.4) \mathrm{E} 16$ & $0.03 \%$ \\
\hline
\end{tabular}

These results confirm prior reports, as in references 9 and 10, which provide estimates for similar elements in the lunar regolith. Based on analysis of lunar samples brought back from Apollo missions, the major elements in the lunar regolith include oxygen, silicon, aluminum, calcium, magnesium, iron, sodium and titanium. In addition, there are trace amounts of other important elements in the regolith including sulfur, phosphorus, carbon, hydrogen, nitrogen, helium, neon, argon, krypton and xenon as reported in reference 9. All these reports show that the Moon 
has a wide range of resources. Furthermore these lunar resources can be exploited through ISRU capabilities to build infrastructure on the Moon and/or deliver to cis-lunar space for use in space exploration architectures to other solar system destinations.

There have been several studies, as cited previously in references 9-13, examining the opportunities and challenges for developing ISRU capabilities and performing ISRU operations on the Moon. It is generally agreed that it would be very beneficial to develop ISRU capabilities to learn to "live off the land" for future human exploration missions and eventual colonization. If we are to explore other worlds, advanced planning tells us that it is best to take advantage of the resources existent in other worlds instead of transporting all the resources and consumables needed for such long and distant journeys. Being capable of relying on critical ISRU capabilities for any human exploration concept can have several potential impacts to mission design, mission performance, mission assurance and life-cycle costs. In addition, ISRU operations have the potential for spawning numerous opportunities for commercial involvement and lunar markets, thus creating a cis-lunar economy capable of sustaining itself.

However, before ISRU capabilities and operations can flourish, there needs to be extensive prospecting for resources, testing and experiments conducted on the lunar surface to determine if ISRU in reality can be economically feasible. Initially, ground truth data must be obtained to determine the properties of the lunar regolith, location and distribution of volatiles, quantities of hydrogen and water, and accessibility of resources. From the scientific data obtained to date, all indications are that there are vast amounts of water in the lunar poles. The PSR of the poles may be very challenging to operate in. Some of these challenges include illumination, temperature ranges, rough terrain and direct communications to Earth for robotic operations. There will need to be several missions to various sites near the poles and the equators to prospect for resources and identify the hazards to access these resources.

To date, there have not been any ISRU experiments or tests performed on the Moon or any other solar system body. Currently, there are projects in development including Resource Prospector Mission ${ }^{22}$ (RPM) and Mars Oxygen ISRU Experiment ${ }^{23}$ (MOXIE) that plan to test different techniques for oxygen extraction on the Moon and Mars, respectively. More specifically, RPM plans to land a rover in the vicinity of the lunar poles and traverse up to $1 \mathrm{~km}$ prospecting and drilling for resources. RPM plans to carry the Regolith \& Environment Science and Oxygen \& Lunar Volatile Extraction ${ }^{24}$ (RESOLVE) payload consisting of a number of instruments to determine the type and quantity of volatiles and then heat the samples to make water. This will be a challenging mission and critical first step for developing and demonstrating ISRU capabilities on the Moon. Currently, RPM is in the formulation phase with tentative plans for launch by 2019 as stated in reference 22 .

\section{Best Practices from COTS/CRS Programs}

Before launching into description of a potential Lunar COTS program, it is essential to review best practices from the COTS and CRS programs to understand its acquisition approach, its benefits and challenges and how to apply it to a new program.

The COTS Program was formulated by a very small team (including 2 of the authors) at NASA Headquarters' Exploration Systems Mission Directorate in 2005. The goal of this formulation phase was to develop an alternate commercial option to stimulate industry to develop low-cost, ISS crew and cargo transportation services, in parallel with the traditional acquisition efforts that were currently underway for the Ares I and Crew Exploration Vehicle projects for ISS under the Constellation Program. This alternate option focused on taking advantage of a "nontraditional" acquisition strategy which would leverage NASA's Other Transaction Authority (OTA) under the Space Act as a mechanism to invest resources into the emerging space industry to develop and demonstrate new capabilities. This non-traditional acquisition strategy was a dramatic departure at the time from traditional FARbased procurement contracts, such as cost-plus or firm-fixed price contracts.

The OTA is a contractual instrument but very different from the FAR-based contracts which contain lengthy and in some instances burdensome and restrictive FAR clauses. Instead, the elements and clauses of an OTA are very streamlined and ungoverned by the FAR and have been characterized by starting with a clean sheet of paper and regulated by a set of mutually agreeable terms and conditions. Under this OTA, NASA elected to enter into funded Space Act Agreements (SAAs) with COTS participants to stimulate industry to develop capabilities and demonstrate eventual services needed by NASA. It was clear that this strategy would allow much more flexibility to the emerging space industry participants which were the targeted group, without being encumbered with the full administrative and reporting requirements traditionally required by FAR contracts.

Although NASA would have less technical oversight and reviews using this approach, the programmatic and technical risks were reduced by employing pre-negotiated, pay-on-performance milestones within the SAAs. Using this strategy, NASA would only provide funding for the participants after successful completion of performance or 
financial-based milestones ensuring deliberate progress being made towards final demonstration of the capability in the flight environment. Also under this strategy, NASA was not expected to fund the total cost of development since in the final outcome NASA would not own the intellectual property rights nor own or operate the service(s). Instead the COTS participant or commercial space provider would be expected to raise its own investment funds and share the development costs with NASA. Therefore the SAAs were, in essence, establishing commercial space partnerships which were defined as mutually beneficial relationships where both parties make a strategic and resource investment to jointly share cost, development and/or operational risk to create new capabilities or services for mutual benefit.

Another important tactic used in the COTS solicitation was to require the commercial space providers to include feasible and economical business plans in their proposals. It was expected that the providers would be targeting markets outside of NASA and not solely rely on the government to close their business case. This tactic was used to force the providers to develop low-cost capabilities to capture new markets, expand their customer base and create new industries to further commercialize LEO space.

Also from the onset of COTS, it was planned that multiple providers should be selected to increase competition and reduce risk to the program. Since this was a high-risk, high-payoff acquisition approach, it was important to have multiple providers in case one failed to meet its milestones, then it could quickly be replaced by another provider without much penalty in contractual fees. This was another important advantage to using SAAs over FARbased contracts. The SAAs offered multiple off-ramps through the use of its performance-based milestones. If too many milestones were missed making evident that not enough progress was being made, then NASA could terminate the agreement without much penalty, thus reducing risk to the program.

During implementation of COTS, this exact scenario turned out to be the case for one of its participants, Rocketplane-Kistler (RpK). In 2006, two participants, SpaceX and RpK were selected to enter into SAAs with NASA under COTS. Early on in the development phase, RpK missed a few financial-based milestones which indicated to NASA their slow progress in raising the investment funds needed to reach their financial goals. After intense discussion with RpK and close inspection of their financial investment efforts, it was evident that RpK did not have a viable chance of raising the required funding. Therefore, COTS decided to terminate the SAA with RpK in 2007. They then quickly released another solicitation to select another participant to enter into a COTS SAA. As a result, Orbital Sciences Corp was selected in 2008 and successfully completed all its milestones with its final cargo transportation demonstration flight to ISS in 2013.

Another important decision made during implementation of COTS was to award early on the ISS Commercial Resupply Services (CRS) contracts in 2008. The COTS formulation team originally planned for two phases leading to the eventual goal of NASA procuring low-cost orbital transportation services to transport pressurized cargo to LEO to meet NASA's missions. The first phase would culminate in a demonstration of orbital transportation of pressurized cargo to LEO which was implemented during the COTS program. The second phase would procure these cargo transportation services through a traditional FAR-based services contract, named the Commercial Resupply Services contract which were issued by the ISS Program Office. The original plan included granting these service awards after successful demonstration of cargo transportation capabilities to reduce risk to the program. However, based on the commercial provider's schedule for completing COTS and with the anticipated retirement of the Shuttle looming, there was an urgency to award these contracts sooner than later to close the gap within the ISS servicing schedule. Also the providers would need sufficient lead time after contracts were awarded to procure longlead items for their launch vehicles. Therefore, the decision was made to award the CRS contracts much sooner than originally planned. In retrospect, this decision helped make the first phase, COTS, a success as it showed a firm commitment by NASA to procure long-term cargo transportation services ensuring its COTS participants and investors a firm anchor customer in NASA.

As a result of the COTS and CRS programs, 2 new launch vehicles and spacecraft were developed and have been successfully servicing the ISS program with cargo transportation missions: 1)SpaceX's Falcon 9 rocket and Dragon spacecraft; and 2)Orbital's Antares rocket and Cygnus spacecraft. Furthermore, SpaceX has revolutionized the global space transportation industry by significantly reducing space transportation costs for medium-class launch vehicles which is causing intense competition among the other launch vehicle providers. SpaceX also has plans to make the core stage of its Falcon 9 rocket reusable which if successful will further reduce its launch vehicle costs by a factor of 2 or 3 .

The COTS and CRS programs proved to be very successful by implementing a very effective model to lower upfront development and operations costs of space transportation and in-space systems. A recent study ${ }^{25}$ conducted by NASA compared SpaceX's actual development costs for the Falcon 9 (launch vehicle only) to estimated costs using traditional cost-plus methods under FAR. Using the NASA-AF Cost Model (NAFCOM), this study estimated that it would have cost NASA approximately $\$ 4$ Billion to develop the same space transportation system using traditional 
cost-plus contracting methods. The actual development cost to NASA for the SpaceX Falcon 9 and Dragon was $\$ 443$ million $^{26}$, of which only $\$ 200 \mathrm{M}$ were for the Falcon 9 launch vehicle. This is a 2000\% (or 20-to-1) reduction in costs over NASA's estimated cost for the traditional approach. Even given the company partners private investment, estimated as another $\$ 200 \mathrm{M}$ for both launch vehicle and spacecraft, these metrics emphasize the dramatic affordability improvements possible using a commercial partnership approach.

In summary, there are specific attributes or best practices of the COTS model which helped make it an impressive, cost-savings success. These best practices are summarized here again to emphasize their importance:

1) NASA and commercial partners share cost, development and operational risk to demonstrate new capabilities for mutual benefit.

2) NASA makes long-term commitments to procure commercial services to help secure private investments.

3) NASA encourages commercial partners to target other markets outside Government to make their business case close. NASA is anchor customer but not sole customer.

4) NASA uses SAA's to enter into partnership with commercial partners to offer maximum flexibility in design solutions without the full demands and requirements of typical FAR-based contracts.

5) NASA includes pay-on-performance milestones in SAA's to provide several off-ramps and reduce programmatic risk.

6) Commercial partners retain Intellectual Property (IP) rights and operates and owns final product(s).

\section{Proposed Lunar Commercial Orbital Transfer Services (LCOTS) Program}

In order to take advantage of the benefits of utilizing lunar resources, without encumbering NASA with the full cost and risk of developing these resources, the authors recommend to initiate an LCOTS program to enter into commercial space partnerships with industry, to share cost, development and operational risk and to incentivize industry to stimulate a cis-lunar economy. Thus the goal of an LCOTS program is to establish affordable, cis-lunar capabilities and services for mutual benefit. As with COTS, it is recommended that OTAs be leveraged and Space Act Agreements be used as the contracting mechanism between NASA and industry. As proven as a successful method with COTS, SAA's offer industry the maximum flexibility to arrive at a design solution to meet the desired requirements. It also reduces programmatic and development risk to NASA as negotiated milestones are included in the SAA's providing numerous off-ramps to NASA if milestones are not met. Further, as shown in the previous discussion, it is imperative to include in this acquisition strategy firm commitments by NASA to procure long-term, cis-lunar commercial services to guarantee industry an anchor customer to help close their business case. Following this COTS-like acquisition strategy, LCOTS will also encourage industry to target other markets including emerging new markets to make their business cases close so not to be solely dependent on its anchor customer. As the COTS program showed, this strategy in the long-term will dramatically improve affordability and push industry to create new markets and open new businesses thus creating a thriving cis-lunar economy which can eventually lead to an economical and sustainable pathway to Mars.

The primary objectives of the proposed LCOTS program are to 1) demonstrate and enter into operation cis-lunar capabilities; 2) reduce technical and operational risk to Mars mission concepts; and 3) reduce life-cycle costs to Mars architectures. We envision the cis-lunar capabilities to include autonomous lunar landings, robotic prospecting for resources, extraction or mining of lunar resources, drilling for water beneath the surface and ISRU production of propellants, such as, LOX and LH2. In addition, cis-lunar infrastructure will need to be established to enable costeffective commercial services to emerge such as, nuclear or solar power plants, communication and navigation satellites, durable habitats for long-term crew stay, ISRU plants, reusable lunar landers to deliver lunar resources to cis-lunar space and propellant depots at cis-lunar space destinations, such as, Earth-Moon L1 or L2 or Lunar Distant Retrograde Orbits (LDRO).

By partnering with industry to develop and demonstrate these capabilities, it will not only stimulate a cis-lunar economy but it will also reduce the technical and operational risk of developing similar capabilities needed for a Mars architecture. As discussed in Section II, the DRA5 architecture is dependent on ISRU capabilities to create the necessary propellant on the Mars surface for the return trip home. Also learning how to "live off the land" through ISRU activities, tele-operation of robotic systems, and development of long-term life-support systems will all be very beneficial to long-term human missions to the surface of Mars.

To meet the third objective of an LCOTS program, i.e. to reduce life-cycle costs, it is important for NASA to show in the early stages of the program its intent of awarding long-term contracts for cis-lunar commercial services. If these services are well-aligned and integrated into the plans for a Mars architecture, then these services will be programmed to enable a Mars transportation system and will directly impact the life-cycle costs of such a system. 
An important example of a commercial service is delivery of lunar-derived propellant (LOX/LH2) to a propellant depot at a cis-lunar space destination. As was shown in Section II, approximately 50 to $60 \%$ of the IMLEO needed for a typical Mars transfer vehicle is propellant. Therefore, if propellant at a cost-effective rate can be retrieved from a cis-lunar propellant depot than it would reduce the number of heavy-lift launches needed from Earth and enable more options for hardware to be accommodated on its scheduled launches. As will be shown in Section VIII, an economic assessment of such a strategy proves to be cost-effective to a Mars architecture and beneficial to stimulating a cis-lunar economy.

\section{A. Phased-Development Approach for a Potential LCOTS Program}

It will be beneficial to develop the LCOTS program in a phased-development approach. This approach will allow for incremental development and demonstration of capabilities before committing to the next phase. This is also a lower-risk approach since it allows for several off-ramps to the program and time for pause between phases to make sure its milestones and objectives are being met before proceeding to the next phase. This approach will also allow for a modest funding commitment to initiate Phase I to determine if sufficient industry interest and financial investors materialize as planned.

As discussed previously, the preferred contractual mechanism for entering into partnerships with industry under LCOTS will be SAA's. A phased-development approach will work very well together with the SAA's as it will allow for well-conceived, achievable milestones in each SAA for each phase. To further reduce risk to the program, it is recommended to include several hardware-demonstration and financial milestones integrated well with the schedule development plan to track and demonstrate progress throughout each phase. If milestones are not achieved in a timely manner according to plan, then it will provide several opportunities to re-negotiate milestones if necessary, and/or terminate partnership agreement if not enough progress is being made. These are all built-in mechanisms to the LCOTS program to reduce risk as much as possible to all parties and to ensure that objectives for each phase are being met as planned. The following sub-sections describe the proposed objectives for each phase.

\section{Phase I - Surfaces Resources and Hazards Assessment}

The goal for the first phase of LCOTS is to assess surface resources and hazards data at various lunar sites by examining ground truth and reconnaissance data provided by lunar rovers and instrument packages sent to the lunar surface. It is important to make these assessment in the initial phase to determine economic viability of extracting these resources given the hazards associated with each location. Some of the hazards that may impact economic viability include difficult landing conditions, limited sun illumination, rocky or steep terrain, extreme temperature environments, limited direct communications to Earth which would inhibit tele-operations of robotic systems. Also, the types and quantities of easily-accessible resources may vary from site to site.

As discussed in Section III, there are a wide variety of resources in the lunar regolith but ground truth data is needed to determine the specific amounts and precise locations for these resources before committing significant investment funds to begin ISRU operations. Therefore, it is imperative to gather sufficient ground truth data at several sites including the lunar poles and along the equator to perform a comparative, economic analysis that will lead to an informed decision on the best economical approach for resource extraction before entering the next phase, Lunar ISRU demonstration.

To achieve the goals of Phase I, the following specific objectives are proposed to not only obtain surface resources and hazards assessment data but also to demonstrate critical capabilities, such as lunar cargo transportation and resource extraction that meet specific requirements, such as, precise landing and energy-efficient resource extraction. Successful demonstration of these capabilities and ability to meet the requirements will determine if there is enough potential and justification to move on to the second phase of the program.

Below is the list of Phase I objectives to be met before entering Phase II of the LCOTS program.

1) Demonstrate capabilities to transport payloads from Earth to Lunar Surface cost effectively.

2) Prospect several sites for surface resources and hazards data:

a. Provide ground truth data at various sites including volatiles, water-ice deposits, lunar regolith properties, thermal environments, etc.

b. Assess potential sites for hazards and accessibility, such as, rough terrain, rocky surface, steep hills, etc.

3) Demonstrate capabilities for lunar resource extraction and/or potential ISRU operations.

\section{Phase II - Lunar ISRU Demonstration}

The goal for Phase II is to continue maturing and demonstrating capabilities to reduce technical and financial risk before entering Phase III which will award long-term services agreement for ISRU production and delivery to cis-lunar destinations. The particular type of capabilities to be demonstrated will depend upon the partner's proposed 
technical development and business plan for providing commercial services. In general these capabilities may range from $\mathrm{H} 2 \mathrm{O}$ extraction from the poles to cracking the $\mathrm{H} 2 \mathrm{O}$ to produce LOX and LH2. These capabilities must be matured enough to demonstrate sufficient reliability, maintainability and affordability to demonstrate the feasibility to scale up production and achieve an economically, viable business plan.

In addition, there may be several capabilities that support an infrastructure needed to enable the commercial services, such as, communications and navigation satellites and solar or nuclear power plants. These capabilities may be just as important as the ISRU production capabilities to ensure an economical plan. It will be important to make a full assessment of the partner's plans for providing commercial services before reaching agreement on which capabilities will need to be demonstrated to reduce risk before proceeding to the next phase.

The specific objectives in support of the goals of the second phase are listed as follows:

1) Demonstrate capabilities for ISRU resource production, such as $\mathrm{H} 2 \mathrm{O}, \mathrm{LOX}, \mathrm{LH} 2$, and storage on a pilot scale program (1 or $2 \mathrm{mt}$ of resource).

2) Demonstrate feasibility and economics of scaling up production and capability to store up to several tons of resources on lunar surface.

3) Demonstrate capability to transport large payloads from lunar surface to cis-lunar space (Earth-Moon Lagrange points or Lunar Distant Retrograde Orbit) for long-term storage

Again, it is recommended that hardware-demonstration and financial milestones be negotiated and included in the SAA's of Phase II to reduce programmatic risk to LCOTS by providing several off-ramps to the program. If there are too many high-risk capabilities that need to be demonstrated, Phase II can be further broken down into several sub-parts to ensure a realistic, schedule development and budget plan are achieved.

\section{Phase III - Lunar ISRU Production and Delivery Services}

The proposed third phase of LCOTS is very analogous to the ISS Commercial Resupply Services program where it awarded long-term service agreements to multiple industry partners after successful demonstration of critical capabilities specified under COTS. The plan for the third phase of LCOTS will follow this same strategy. After successful demonstrations in Phases I and II, the third phase will award long-term service agreements to multiple industry partners, if possible, to provide essential services in support of a Mars transportation system, such as inspace re-fueling services. Therefore, the specific objectives in support of the goals of the third phase are as follows:

1) NASA awards long-term contract for Lunar ISRU production of $\mathrm{H} 2 \mathrm{O}$ or $\mathrm{LOX} / \mathrm{LH} 2$ on the order of several tons per year.

2) Awards are also made for delivery services to Cis-Lunar Propellant Depot.

3) Awards are made to multiple commercial providers to reduce risk and enable competition.

\section{Maturity Assessment of Candidate Capabilities for LCOTS}

Before initiating an LCOTS program, a careful assessment should be performed to determine the capabilities that are at a high technical maturity level to be successfully demonstrated by industry under a COTS-like model. Other important factors to consider during this assessment are: 1) number of viable companies with enough technical and financial capability and strong interest to pursue LCOTS opportunity; 2) size of potential markets likely to emerge within 5 years to attract private investors; 3 ) level of affordability to fully develop capability within realistic budgets from NASA and private capital from industry; 4) strong potential for positive return on investment based on sound business plans; and 5) strong potential to reduce technical, cost or operational risk towards a Mars architecture.

During the formulation phase of the COTS program in 2005, a similar assessment was carefully performed over a 6 to 8 month period to determine industry interest and readiness to demonstrate various capabilities given a realistic program budget. In the beginning, there was a strong push for commercial crew capability to be demonstrated in the first phase of COTS. However, after careful study and numerous interviews with potential industry partners, it was determined that this approach would be burdened with too high technical, cost and safety risk to pursue at the time. A more reasonable approach was taken to demonstrate unpressurized and pressurized cargo capabilities to ISS for COTS. This approach would still result in essential capabilities needed for ISS operations, however, with a much lower technical and financial risk profile. Therefore, the final recommendation to the NASA Administrator was to initiate the COTS program to primarily pursue unpressurized and pressurized cargo capabilities to meet the cargo requirements of the ISS which turned out to be a very successful program as previously discussed. It was only after successful completion of the COTS program that the commercial crew 
program was initiated with several risk reduction phases initially to mature capability and reduce technical and financial risk.

Similarly, an LCOTS program should conduct a careful assessment of capabilities to be demonstrated in each of its phases to increase likelihood of success for the program. A preliminary assessment was conducted using the criteria of Table 4. These criteria were established by the authors from lessons learned and insight gained from firsthand experience working on the COTS program. This table provides a description of each criteria and a defined range for high, medium and low ratings for this assessment. These criteria are important metrics to help decide if a capability is ready to be developed and demonstrated using a COTS-like approach or if a more traditional FARbased approach is recommended due to the estimated risk. It is also important to consider at this point if the longterm capability or service should be commercially or government-owned and operated. The advantages and disadvantages of these different models should be discussed and carefully weighed before making final decision on the capabilities and/or services for a COTS-like demonstration.

Table 4. Criteria for Assessing LCOTS Capabilities for Phases I and II

\begin{tabular}{|c|c|c|}
\hline Criteria & Description & Hi/Med/Lo Definitions \\
\hline Industry Maturity/Capability & $\begin{array}{l}\text { Readiness or maturity level of industry capability } \\
\text { to perform successfully in an operational space } \\
\text { environment. }\end{array}$ & $\begin{array}{l}\text { High }- \text { TRL } 6 \text { or above } \\
\text { Med - TRL } 4 \text { or } 5 \\
\text { Low - TRL } 3 \text { and below }\end{array}$ \\
\hline Viable Companies & $\begin{array}{l}\text { Number of viable companies that exemplify } \\
\text { strong financial and technical capabilities and } \\
\text { ability to raise significant investment funds for } \\
\text { proposed capability demonstration. }\end{array}$ & $\begin{array}{l}\text { High }-3 \text { or more companies } \\
\text { Med }-1 \text { or } 2 \text { companies } \\
\text { Low }-0\end{array}$ \\
\hline Significant Market & $\begin{array}{l}\text { Measure of potential for emergence of near-term } \\
\text { markets (within } 5 \text { years) beyond NASA's needs } \\
\text { to enable cost sharing (private financing) and } \\
\text { cost-effective pricing. }\end{array}$ & $\begin{array}{l}\frac{\text { High }}{\text { next } 5 \text { years }} \$ 500 \mathrm{M} \text { markets in } \\
\text { Med }- \text { Between } \$ 100 \mathrm{M} \text { to } \\
\$ 500 \mathrm{M} \text { markets in next } 5 \text { years } \\
\underline{\text { Low }- \text { Emergence of markets in }} \\
\text { over } 5 \text { years }\end{array}$ \\
\hline $\begin{array}{l}\text { Positive Return on Investment } \\
\text { (ROI) }\end{array}$ & $\begin{array}{l}\text { Measure of potential for positive ROI includes } \\
\text { level of affordability to fully develop capability, } \\
\text { proposed price point for capability and overall } \\
\text { business plan to achieve ROI. }\end{array}$ & 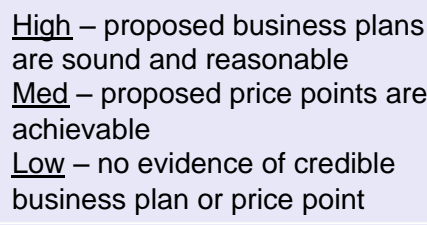 \\
\hline Risk Reduction for Mars & $\begin{array}{l}\text { Measure of potential to reduce technical, } \\
\text { operational or cost risk for capability that may be } \\
\text { a critical element of a Mars architecture. }\end{array}$ & 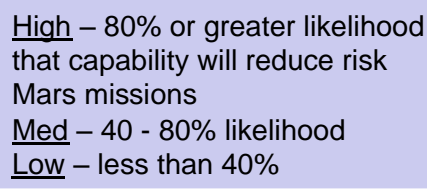 \\
\hline
\end{tabular}

After the criteria were set, a list of candidate capabilities was then compiled to meet the objectives of Phase I and II. These candidate capabilities were then down-selected to the top capabilities that would greatly benefit from development within a commercial space partnership. Figure 5 provides a list of these top capabilities which are divided between Phases I and II as shown in the figure. This figure also shows the preliminary assessments for each capability measured against the corresponding criteria as distinguished by the high, medium, and low colored boxes.

To enable these assessments, it was important to gather information on the viable companies from the emerging space industry showing strong interest in developing cis-lunar capabilities. In recent years, there have emerged a growing number of these companies that with business plans to conduct operations and provide services in the vicinity or surface of the Moon. These companies are listed in Table 5. The data in this table was obtained from public websites and publically available sources. The companies listed in this table are a representative list and not meant to be exhaustive. In the follow-up work to this study, this list will be updated from interviews and further research into these companies. Companies not on this list are encouraged to contact the authors to participate in this analysis. 


\begin{tabular}{|c|c|c|c|c|c|c|}
\hline & Capability-Description & $\begin{array}{l}\text { Industry } \\
\text { Capability/ } \\
\text { Maturity }\end{array}$ & $\begin{array}{c}\text { Viable } \\
\text { Companies }\end{array}$ & $\begin{array}{l}\text { Significant Market } \\
\text { Beyond NASA }\end{array}$ & $\begin{array}{l}\text { Potential for } \\
\text { Positive ROI }\end{array}$ & $\begin{array}{l}\text { Risk Reduction } \\
\text { for Mars }\end{array}$ \\
\hline \multirow{4}{*}{ 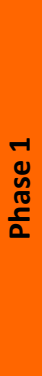 } & $\begin{array}{l}\text { Lunar Landers - Delivers } \\
\text { payload < } 1 \mathrm{mt} \text { to surface }\end{array}$ & High & High & High & High & Medium \\
\hline & $\begin{array}{l}\text { Lunar Rovers - small rovers } \\
\text { for traversing distances up to } \\
50 \mathrm{~km}\end{array}$ & High & High & High & High & Medium \\
\hline & $\begin{array}{l}\text { Instrument package- for } \\
\text { prospecting resources and } \\
\text { identifying hazards }\end{array}$ & Medium & Low & Low & Medium & Medium \\
\hline & $\begin{array}{l}\text { ISRU Demo- H2O or other } \\
\text { resources extraction }\end{array}$ & Medium & Medium & High & Medium & High \\
\hline \multirow{5}{*}{ 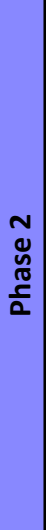 } & $\begin{array}{l}\text { Comm and Navigation } \\
\text { Satellites - to enable large- } \\
\text { scale ISRU operations }\end{array}$ & High & High & High & High & Medium \\
\hline & $\begin{array}{l}\text { Solar or Nuclear Power } \\
\text { Stations - for sunlit or dark } \\
\text { operations }\end{array}$ & Medium & Medium & High & High & High \\
\hline & $\begin{array}{l}\text { Pilot-Scale ISRU } \\
\text { Production - to produce } 1 \text { or } \\
2 \mathrm{mt} \text { of } \mathrm{H} 2 \mathrm{O} \text { or LOX/LH2 }\end{array}$ & Medium & Medium & Medium & Medium & High \\
\hline & $\begin{array}{l}\text { Reusable Lunar Landers - } \\
\text { to deliver large payloads to } \\
\text { propellant depot }\end{array}$ & Low & Low & Low & Low & High \\
\hline & $\begin{array}{l}\text { Propellant Depot in Cis- } \\
\text { Lunar Space - to store } \\
\text { several tons of } \mathrm{H} 2 \mathrm{O} \text { or prop }\end{array}$ & Low & Low & Low & Low & High \\
\hline
\end{tabular}

Figure 5. Preliminary Assessment of Potential Capability Candidates for LCOTS

Table 5. List of Emerging Space Companies and their prospective Business Plans

\begin{tabular}{|l|l|}
\hline Emerging Space Companies & Business Strategy \\
\hline Astrobotic Technology & $\begin{array}{l}\text { Low-cost lunar transportation and rovers to the } \\
\text { surface of the Moon; delivery of lunar payloads on } \\
\text { routine basis. }\end{array}$ \\
\hline Moon Express & $\begin{array}{l}\text { Low-cost robotic missions to the Moon for mining } \\
\text { resources. }\end{array}$ \\
\hline Masten Space Systems & $\begin{array}{l}\text { Vertical Take-Off and Vertical-Landing Rockets; } \\
\text { Lunar Landers }\end{array}$ \\
\hline Bigelow Aerospace & LEO space habitats and lunar habitats \\
\hline Shackleton Energy & $\begin{array}{l}\text { Resource prospecting missions to the Moon. Space- } \\
\text { based solar power. }\end{array}$ \\
\hline Deep Space Industries & Launch of cubesats to scout near-Earth asteroids \\
\hline Planetary Resources & $\begin{array}{l}\text { LEO-based telescope to identify and characterize } \\
\text { asteroids for mining potential. }\end{array}$ \\
\hline Golden Spike & Crew transportation to the Moon and return. \\
\hline
\end{tabular}


From Figure 5, the top 2 capabilities that emerged with mostly high ratings were lunar landers and lunar rovers. These 2 capabilities scored very high because of the number of companies showing strong interest, economical business plans and amount of private investments raised so far. The top 3 companies in this category are listed in Table 5. All 3 of these companies are also participating in NASA's Lunar Cargo Transportation and Landing by Soft Touchdown (CATALYST) Program sponsored by HEOMD which were awarded un-funded Space Act Agreements in 2014 to develop lunar transportation systems. The purpose of the Lunar CATALYST program is to encourage the development of robotic lunar landers that can be integrated with commercial launch vehicles to deliver payloads to the lunar surface.

The other 2 capabilities listed for Phase I, instrument package and ISRU demonstration, should probably be delivered by NASA since there is more experience and technical capability to develop these capabilities within NASA at this time than industry. However, before making final decisions for Phase I, it is recommended that a more detailed assessment be made for the top capabilities of Phase I to more precisely evaluate maturity level, affordability, business plans including markets and potential for positive ROI. All these factors are very important to estimate likelihood of success to demonstrate capability for a given schedule and budget.

For Phase II, the assessments of Figure 5 show that communications and navigation satellites, and solar or nuclear power stations scored mostly high against the criteria. It is very clear that these capabilities are much needed to support any commercial or government-owned operation on the lunar surface. Furthermore, it could be debated that these candidate capabilities should be moved into Phase I instead of delaying development until Phase II. As with most programs, the deciding factor will be the budget limitations for each phase. However, given the goals of Phase I to prospect for resources and identify hazards on the lunar surface, the top 2 capabilities, lunar landers and rovers, should be given top priority for Phase I since you first need to identify the most economical sites for lunar extraction of resources before setting up your power stations and navigation satellites.

The bottom 2 capabilities of Figure 5, reusable lunar landers and propellant depot in cis-lunar space, scored very low in this preliminary assessment. This shows that these capabilities are not ready to be demonstrated by industry at this time. It is recommended that technologies enabling these capabilities, such as cryogenic zero-boiloff and propellant transfer, continue to be matured within NASA.

\section{Implementation Options for LCOTS Phases I and II}

To initiate Phase I of LCOTS, three options are presented to meet the goals and objectives of Phase I and develop the top capabilities recommended from the preliminary assessments described in the previous section. In addition, an option for Phase II is also presented in the section below.

\section{A. Phase I - Option 1}

To meet the objectives of Phase I, multiple landers and rovers will be needed to prospect for resources, identify hazards and determine the most economical and beneficial site for extracting resources on the surface of the Moon. A low-cost approach to obtaining surfaces resources and hazards data would be to take advantage of the progress already made by the commercial space industry who are in current pursuit of Google Lunar X-Prize.

This X-Prize was initiated in 2007 by Google "to incentivize space entrepreneurs to create a new era of affordable access to the Moon and beyond" as stated on their website ${ }^{\mathrm{vi}}$. The competition offers $\$ 30 \mathrm{M}$ in prizes including \$20M Grand Prize to the first team that successfully lands on the Moon, traverses 500 meters and transmits high definition images and video back to Earth. Although the teams must use mainly private funds (90\%) to develop their systems, the guidelines do allow for government to provide or purchase services from the teams at fair market value.

Following these guidelines, a low-cost option for NASA to obtain ground truth data is to purchase payload delivery services from one or more of the teams to deliver a government-furnished instrumentation payload to a desired lunar site. This instrumentation payload could be competed within NASA or selected from external organizations. One option for this payload may be the RESOLVE instrumentation suite which is being developed for NASA's RPM project as discussed earlier in Section III. The RESOLVE payload includes such instruments as a neutron spectrometer system, near-infrared volatiles spectrometer system and lunar advanced volatile analysis to prospect for resources and oxygen \& volatile extraction node to demonstrate ISRU capability. This instrumentation suite can be adjusted and/or distributed to fit on one or more of the lunar X-Prize landers and rovers. Also, since RPM is planned to launch some time in 2019, the Lunar X-Prize flights would complement this mission by delivering reproductions of these mature instruments on additional rovers at various lunar sites.

\footnotetext{
${ }^{\text {vi } h t t p: / / l u n a r . x p r i z e . o r g / a b o u t / o v e r v i e w ~}$
} 
In addition, penetrators or probes similar to those flown on Deep Space 2 mission ${ }^{27}$ may also be released from the landers to penetrate beneath the lunar surface and provide measurements of the volatile concentrations at various sites as well. Together, these instruments and flight platforms can serve to accomplish the goals and objectives of Phase I in partnership with commercial space providers at a reasonable and affordable cost to the government.

Table 6 below summarizes the resources needed for this Lunar Payload Delivery Services option. For this option, it is assumed that a medium-size launch vehicle, such as, Falcon 9 or Atlas V rocket would be sufficient to deliver a rover with instrumentation suite. The estimated mass of the instrumentation suite and rover may reach up to $270 \mathrm{~kg}$. As shown in Table 6, the total estimated cost ranges from $\$ 50 \mathrm{M}$ to $\$ 100 \mathrm{M}$. The range in costs vary depending on the partnership agreement between LCOTS partners (NASA and Lunar X-Prize team) to share cost and risk to develop lunar transportation capability.

The basis for these estimates was the data obtained from one of the Lunar X-Prize teams, Astrobotics. Their payload user guide ${ }^{28}$ claims their Griffin lunar lander can deliver up to $270 \mathrm{~kg}$ to the lunar surface from a Falcon 9 launch starting at $\$ 1.2 \mathrm{M} / \mathrm{kg}$. If NASA takes advantage of the first Lunar X-prize flight, then the lander and rover systems will be furnished by the Lunar X-prize teams. This is a very low-cost approach, however, higher risk is being taken since it will be the initial flight for one of the teams.

Table 6. Option 1 - Lunar Payload Delivery Services

\begin{tabular}{|c|c|c|c|}
\hline $\begin{array}{c}\text { Resources } \\
\text { Needed }\end{array}$ & Requirements & Potential Sources & $\begin{array}{l}\text { Range in Cost for } \\
\text { LCOTS Partners }\end{array}$ \\
\hline $\begin{array}{l}\text { Launch } \\
\text { Vehicle }\end{array}$ & $\begin{array}{l}\text { Launch payload to } \\
\text { LEO and perform TLI } \\
\text { to LLO;. }\end{array}$ & $\begin{array}{l}\text { SpaceX - Falcon } 9 \\
\text { ULA - Atlas V }\end{array}$ & $\$ 30 M$ to $\$ 60 M$ \\
\hline $\begin{array}{l}\text { Landers and } \\
\text { Rovers }\end{array}$ & $\begin{array}{l}\text { Deliver up to } 270 \mathrm{~kg} \\
\text { to lunar surface. }\end{array}$ & $\begin{array}{l}\text { Google Lunar X-Prize } \\
\text { Teams }\end{array}$ & \\
\hline \multirow[t]{2}{*}{$\begin{array}{l}\text { Prospecting } \\
\text { Instruments } \\
\text { and ISRU } \\
\text { demo } \\
\text { equipment }\end{array}$} & $\begin{array}{l}\text { Govt to furnish } \\
\text { instruments and } \\
\text { equipment. }\end{array}$ & $\begin{array}{l}\text { Candidate } \\
\text { instrumentation } \\
\text { package: RESOLVE }\end{array}$ & $\begin{array}{l}\$ 20 M \text { to } \$ 40 M \text { to } \\
\text { deliver instrument } \\
\text { payload }\end{array}$ \\
\hline & & Total Range in Cost & $\$ 50 M$ to $\$ 100 M$ \\
\hline
\end{tabular}

\section{B. Phase I - Option 2: Prospecting and ISRU Demonstration}

As a second option, a heavy-class, launch vehicle, such as Falcon Heavy or ULA's Vulcan rocket, is recommended to be used in partnership with the Google Lunar X-Prize teams to deliver multiple payloads to multiple sites on a single launch. Although this option is at higher cost, it is more advantageous because it offers multiple opportunities to obtain ground truth data in a shorter amount of time than Option 1. This strategy also lowers the risk of mission success by flying multiple missions at once, provided that there is a second set of units for each lander and a backup flight opportunity to protect against a launch failure. The strategy is especially effective for risk reduction and site optimization in the event that the first set is successfully flown and five additional sites are subsequently characterized with the back-up set of hardware.

Although neither the Falcon Heavy nor ULA's Vulcan have launched yet, they are estimated to be operational by 2016 and 2019, respectively. As projected by SpaceX, Falcon Heavy will have payload capability up to $53 \mathrm{mt}$ to LEO at cost-effective pricing of $\$ 1700 / \mathrm{kg}$ as cited on their website ${ }^{\mathrm{vii}}$. Additionally, as reported in the ELA study of reference 11, it is estimated that a single launch of Falcon Heavy can deliver up to 5 lander/rover systems to probable sites near the lunar poles and equator to prospect for resources.

Since ULA's Vulcan rocket was recently announced, there was not sufficient data at the time to conduct similar analysis on this system. However, it is expected that it's powerful upper-stage Advanced Cryogenic Evolved Stage (ACES) "will have greater capability than any other rocket on the market" as cited on their website viii.

For this option, it is proposed that the instruments for prospecting and ISRU demonstration equipment be competed and supplied by commercial industry or government-furnished equipment (GFE) as in Option 1. If there is

\footnotetext{
vii http://www.spacex.com/about/capabilities

viii http://www.ulalaunch.com/Products_Vulcan.aspx
} 
enough lead time for this option, there may be sufficient interest from industry to build and test their prospecting and ISRU instrumentation and equipment. This may prove to be a cost-effective approach since some companies have expressed strong interest in extracting resources from the lunar surface to fit within their business plans as shown in Table 5. Table 7 below summarizes the resources, requirements and approximate range of costs for this Prospecting and ISRU demonstrations. The potential sources listed in this table are meant to be representative and not an exhaustive list. In the forward work, a more comprehensive list will be sought for all the options. In addition, the range in costs vary depending on the partnership agreement between LCOTS partners as mentioned previously to share cost and risk to develop prospecting and ISRU capabilities.

Table 7. Option 2 - Prospecting and ISRU Demonstrations

\begin{tabular}{|c|c|c|c|}
\hline $\begin{array}{l}\text { Resources } \\
\text { Needed }\end{array}$ & Requirements & Potential Sources & $\begin{array}{l}\text { Range in Costs for } \\
\text { LCOTS Partners }\end{array}$ \\
\hline Launch Vehicle & $\begin{array}{l}\text { Launch payload to LEO } \\
\text { and perform TLI to LLO; }\end{array}$ & $\begin{array}{l}\text { SpaceX - Falcon Heavy } \\
\text { ULA - Vulcan Rocket }\end{array}$ & $\$ 50 \mathrm{M}$ to $\$ 90 \mathrm{M}$ \\
\hline $\begin{array}{l}\text { Landers and } \\
\text { Rovers }\end{array}$ & $\begin{array}{l}\text { Deliver up to } 5 \text { lander/ } \\
\text { rover systems to lunar } \\
\text { surface. }\end{array}$ & $\begin{array}{l}\text { Google Lunar X-Prize } \\
\text { Teams }\end{array}$ & \\
\hline \multirow[t]{2}{*}{$\begin{array}{l}\text { Prospecting } \\
\text { and ISRU demo } \\
\text { equipment }\end{array}$} & $\begin{array}{l}\text { Prospecting instruments } \\
\text { to identify resources; } \\
\text { ISRU equipment to demo } \\
\text { capabilities, such as } \mathrm{H} 2 \mathrm{O} \\
\text { extraction. }\end{array}$ & $\begin{array}{l}\text { Shackleton Energy } \\
\text { Moon Express } \\
\text { Or GFE }\end{array}$ & $\begin{array}{l}\$ 20 M \text { to } \$ 30 M \text { for } \\
\text { each payload (up } \\
\text { to } 5 \text { ) }\end{array}$ \\
\hline & & Total Range in Cost & $\$ 150 M$ to $\$ 240 M$ \\
\hline
\end{tabular}

\section{Phase I - Option 3}

A third and final option for Phase I will also demonstrate lunar cargo transportation and ISRU capability. However, this option does not take advantage of the Google Lunar X-Prize competition for several reasons, such as, the competition is completed before Phase I is underway. Presently the Lunar X-Prize deadline for winning the Grand Prize is December 31, 2017.

In this scenario, a heavy-class launch vehicle, similar to option 2, is used to deliver several landers and rovers (up to 5) to the lunar surface. However since the competition is not leveraged, the estimated costs significantly increase compared to option 2 because the costs for the landers and rovers will have to be added into this option which was excluded from option 2. Depending on when this option is initiated, the costs for the landers and rovers will need to be negotiated with the viable suppliers at the time. Also this option will also offer the opportunity for industry demonstration of instruments and ISRU equipment through an LCOTS partnership agreement as previously discussed. Although this third option is offered here as a viable option, more work will be needed to fully develop this option and provide a fair estimate of costs.

\section{Phase II Option for Pilot-Scale H2O Extraction Demonstration}

To meet the goals and objectives of Phase 2, an option for a pilot-scale demonstration of $\mathrm{H} 2 \mathrm{O}$ extraction is presented in Table 8. This option allows for demonstration of robotic operations of ISRU equipment to extract $\mathrm{H} 2 \mathrm{O}$ from water-ice deposits at the poles, then process and store the water up to 1 to $2 \mathrm{mt}$. This pilot-scale operation will demonstrate all steps necessary for full-scale $\mathrm{H} 2 \mathrm{O}$ extraction operation which is necessary to determine feasibility and economics of a full-scale operation.

Table 8 includes several assumptions for this option. It assumes that only 1 heavy-lift launch is necessary to deliver the excavator/hauler (up to $1 \mathrm{mt}$ ) and the $\mathrm{H} 2 \mathrm{O}$ processor and storage tank (up to $1 \mathrm{mt}$ ). It also assumes power stations, such as solar or nuclear power stations, have been set up near the pilot-scale demonstration site and offer the necessary power demands of the ISRU operations. It also assumes communications and navigation satellites have been put in place for polar operations which allow for direct communication to Earth during all robotic operations.

Also the basis of estimate used for Table 8 range of costs were derived from cost estimates provided in the ELA study ${ }^{11}$ and Spudis/Lavoie ${ }^{13}$ conference paper. These studies examined architectures to develop ISRU operational 
plants on the lunar surface capable of delivering up to $200 \mathrm{mt}$ of propellant (LOX/LH2) on a yearly basis to cis-lunar space destinations.

The total estimated cost in Table 8 were approximated based on these full-scale operations and expenses but scaled down proportionately to produce 1 to $2 \mathrm{mt}$ of water. Follow-on work is needed to further refine these estimates and identify a complete list of potential companies that can develop these capabilities and meet the full criteria of Table 4 as previously discussed. It should also be noted that if other lunar industries emerge in the next 5 years, then there will be a significant market for the production of water which will further drive down costs.

Finally, there are many other possible options that can meet the goals and objectives of Phase II. Only one option is presented here to show order of magnitude costs for completing Phase II. A more feasible option can be developed after Phase I is underway to more accurately determine industry capability, investment opportunities and emerging markets which will all have an impact on estimated costs and timeline.

Table 8. Phase 2 Option Estimated Expenses

\begin{tabular}{|c|c|c|}
\hline $\begin{array}{l}\text { Resources } \\
\text { Needed }\end{array}$ & Description & $\begin{array}{l}\text { Representative } \\
\text { Companies }\end{array}$ \\
\hline Launch Vehicle & $\begin{array}{l}\text { Launch payload to LEO and } \\
\text { perform TLI to LLO }\end{array}$ & $\begin{array}{l}\text { SpaceX - Falcon Heavy } \\
\text { ULA - Vulcan Rocket }\end{array}$ \\
\hline Lunar Landers & $\begin{array}{l}\text { Transport payloads (up to } 2 \mathrm{mt} \text { ) } \\
\text { from LLO to Lunar Poles }\end{array}$ & $\begin{array}{l}\text { Google Lunar X-Prize } \\
\text { Teams }\end{array}$ \\
\hline Excavator/Hauler & $\begin{array}{l}\text { Medium-size rover(up to } 1 \mathrm{mt} \text { ) to } \\
\text { dig and move lunar soil from } \\
\text { polar ice deposits to water } \\
\text { processors; }\end{array}$ & Caterpillar \\
\hline \multirow[t]{2}{*}{$\begin{array}{l}\mathrm{H} 2 \mathrm{O} \text { Processing } \\
\text { and Storage Tanks }\end{array}$} & $\begin{array}{l}\text { Demo-size processor and tank (up } \\
\text { to } 1 \mathrm{mt} \text { ) capable of storing up to } 2 \\
\mathrm{mt} \text { of water }\end{array}$ & Shackleton Energy \\
\hline & Total Estimated Cost & $\$ 350 M$ to $\$ 400 M$ \\
\hline
\end{tabular}

\section{Economic Assessment of Lunar Propellant for Mars Mission}

Economic assessments of the proposition of lunar propellant for human Mars missions conducted via business cases and/or NASA budget analysis often mixes private and public funds according to approach. A "what-if" business case analysis may involve NASA paying for most or all up-front costs ${ }^{29}$. While major cost elements for developing and establishing a lunar ISRU capability, such as, ISRU plant, propellant depot, etc, may appear manageable, establishing the capability as a whole can amount to some tens of billions of dollars ${ }^{11,13}$-especially if including human tending as these architectures recommend. As an example, the recent ELA study resulted in estimated costs of \$40B over a 10-12 year period to establish a permanent commercial lunar base that can create and deliver up to $200 \mathrm{mt}$ of propellant per year to cis-lunar space. Although this may appear as a fairly high investment, a fair economic assessment should also estimate the potential revenue stream against operational costs as well as other economic benefits.

Once operational, a revenue stream sensitivity is often assessed around some price per $\mathrm{kg}$. A revenue of $\$ 10,000$ per kg has been considered as a price NASA might pay for propellant since "the value of fuel for a single Mars mission may be several billion dollars by itself" ${ }^{\prime 30}$. Combined with Mars mission needs of $650 \mathrm{mt}$ of propellant or more as discussed in Section II, it becomes evident that there is a potential multi-billion dollar a year revenue opportunity.

Approaching the business case viability for lunar propellant can be seen in simpler terms as the process by which an effort on Earth, and a related space activity or interim outcome, is converted finally into delivered propellant. Assuming the propellant is some total kg per year of LOX and LH2, and focusing on the narrow "anchor" customer being NASA, provided that propellant at an interface, the question becomes how possible is it that efforts which cost 
some $\$ / \mathrm{kg}$ get converted to delivered propellant at another $\$ / \mathrm{kg}$ ? This simplification requires understanding the initial efforts on a $\$ / \mathrm{kg}$ basis before these translate into the final product - delivered propellant.

Two key factors that bound the question of turning an effort at some cost into propellant sellable at a price that is higher, adding value to something less expensive than the going price of the finished product, are: an ISRU capabilities autonomy and life limits. To the degree the autonomy of an ISRU operation is high or low, or the life of the in-space infrastructure is high or low, a higher or lower degree of human-tended activity gets integrated into the business equation as a cost.

At one extreme, permanent human tending entails permanent cargo support, habitation, and so on, adding further costs into a lunar propellant business equation. At the other extreme, little or no human tending for propellant production through delivery will have recurring costs driven by the effort to manage, operate and maintain assets such as an ISRU plant/system, reusable landers as tankers, and the depot in lunar orbit, via tele-operation from Earth.

The value to this simplification, of thinking in terms of an effort (cost) converted into propellant (product), is in seeing that an ISRU operation on the Moon can be bounded by the contributions of lower or higher cost efforts which themselves have a cost $/ \mathrm{kg}$ basis. Knowing that in-space crew activity is a cost at well over $\$ 10,000 / \mathrm{kg}$ (a sum total of launcher, spacecraft, etc.) even in the best case today ${ }^{31}$, it would not be possible to have such a cost dominate in a business case pretending to convert such activity into a product sold at only $\$ 10,000 / \mathrm{kg}$. This is the allure of LEO depots ${ }^{32}$, taking launchers and tanker stages at costs less than the $\$ 10,000 / \mathrm{kg}$ propellant price point. Converting a $\$ 2,000$ to $\$ 5,000 / \mathrm{kg}$ cost $^{\mathrm{ix}}$ (launcher payload, tankers, ops) into a $\$ 10,000 / \mathrm{kg}$ final product has immediate appeal.

For a similar propellant delivery concept, but via a depot in cis-lunar space, serviced from a plant on the lunar surface, there is additional potential for needing human tending, not just at the depot in space, but at the facilities on the Moon. Figure 6 compares revenue rates (black dashed lines) versus costs at different rates depending on the level of autonomy and human tending. A more autonomous system will presumably require less human tending and result in lower cost as indicated by the green line in the figure (cost of $\$ 6500 / \mathrm{kg}$ which was scaled appropriately based on the ELA study $\left.{ }^{11}\right)$. Hence, the business case may or may not close depending on the amount of human tending needed. For a modest level of automation, an ISRU lunar propellant operation that requires occasional human tending can close its business case (green line) - if the expensive human tending cost does not overwhelm converting other less expensive costs (efforts) into a total product at less than its selling price point $(\$ 10,000 / \mathrm{kg}$ or as the case may be).

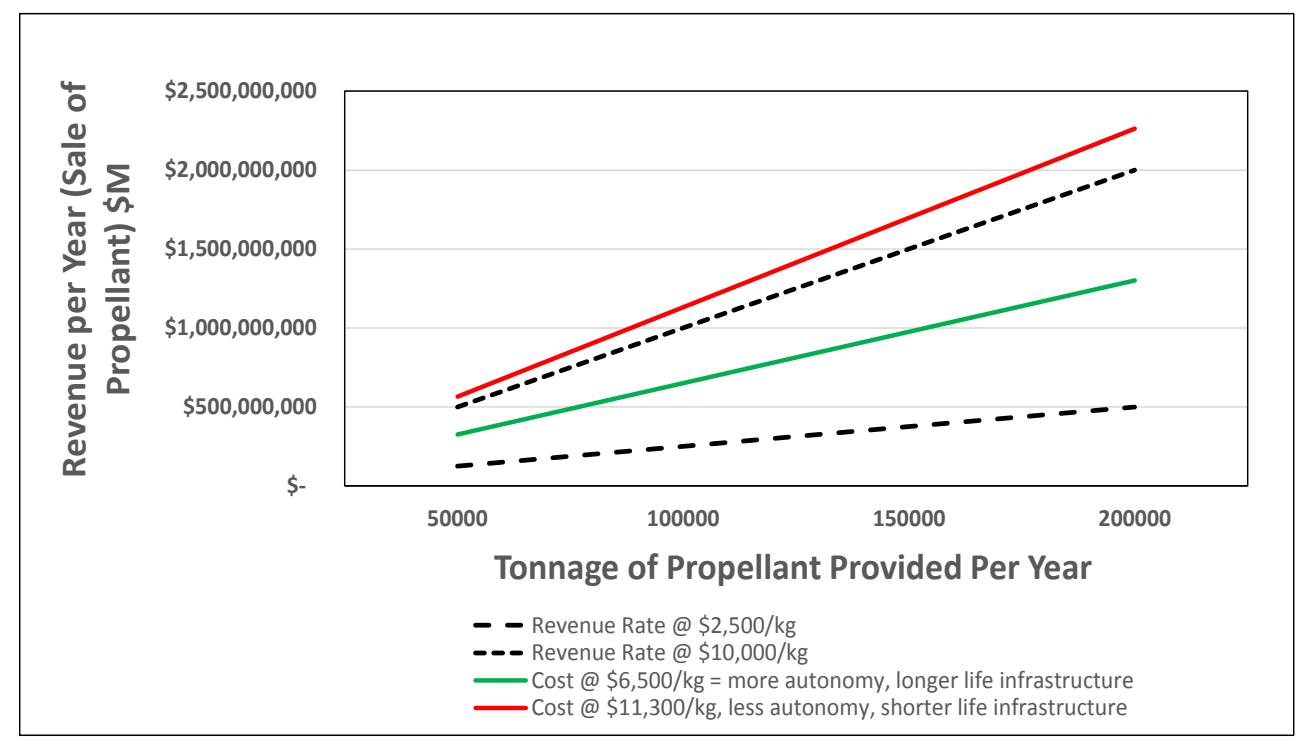

Figure 6. Degrees of autonomy and life limits in propellant production and delivery. These degrees entail more or less crew and related support in determining the business case for lunar propellant for NASA's Mars missions.

\footnotetext{
ix A Falcon 9, consisting of rocket stages and rocket engines, already launches payloads commercially at a cost per usable payload of approximate $\$ 5,000$ per $\mathrm{kg}$. If stages and engines and their effort and systems (flight and ground) can already be sold profitably at this rate, an additional stage acting as a propellant tanker would by extension be possible at a similar rate. Although cumulative, a scale at the Falcon Heavy end, potentially at $\$ 2,000 / \mathrm{kg}$, with the additional tanker atop, would easily accumulate no more than $\$ 4,000 / \mathrm{kg}$ in costs for propellant.
}

American Institute of Aeronautics and Astronautics 
Alternately, a lunar crew for other purposes, not entirely attributable and dedicated to the ISRU operation, would advance the lunar ISRU case even further - amortizing into the ISRU business case only some portion of this cost. Similarly, according to life limits, infrastructure that is tended to at one rate but used over some other Mars mission rate, might advance the business case by amortizing infrastructure operating costs over a number of Mars missions.

Additional amortization of any given costs could occur through productivity - assuming a ready customer for more propellant and that costs continue to be bounded by the basic parameters described previously. Additional revenue against a given cost could arise from many emerging or potential markets as outlined in other studies ${ }^{33}$. Although the market projections in this USC study are preliminary, it emphasizes the importance of maturing such market studies to better estimate potential services and annual income from capabilities developed on the Moon.

Therefore development costs for ISRU and propellant production on the Moon for Mars missions should be seen in balance against its many economic and other benefits. The direct potential economic benefits of lunar ISRU capability are lower life-cycle costs to human Mars missions relative to other options as previously discussed in earlier sections. Other economic benefits may include new revenue streams from new lunar industries, such as, lunar mining, solar power satellites and lunar tourism as discussed in the USC study, reference 33.

Economic efficiency has already been shown in the ELA study of reference 11, whereby capability and infrastructure that is formed around a commercial partnership approach can reduce the costs of the lunar ISRU and lunar depot propellant to levels that are more affordable within the context of NASA budget constraints. Economic efficiency measured by extensibility, the ability of a NASA initiative to create wholly new industries serving nongovernment markets, also furthers other economic goals. Economic growth that is more visible will be able to justify and sustain further NASA investment. Also, market growth beyond NASA would enable products and services that NASA needs for Mars missions at reduced costs to the degree non-government markets provide private investment, maturation of technology, and the opportunity to amortize private business cases over more users.

Performance efficiency as a benefit has also been shown as a possibility using metrics such as IMLEO. Considering a lunar ISRU plant and its tankers and orbital depot capabilities as masses that can be traded against the mass of the propellant required for the same Mars mission, significantly less mass (up to a 48\% IMLEO reduction) ${ }^{34}$ may result from the lunar ISRU route.

Additionally, a lunar ISRU capability would certainly result in technical and operational risk reduction for a future human Mars mission. There would be several technologies and operations matured through this process that would directly benefit a Mars mission, such as, autonomous ISRU operations, tele-operation of robotic systems, long-lasting life support systems, extended crew operations, and surface habitats.

All these benefits should be weighted carefully against the up-front investments needed for a lunar ISRU plant versus an Earth-delivered propellant depot. In addition, the ISRU plant and all related capability (the orbital depot, tankers, etc.) can be seen as competing with $650 \mathrm{mt}$ or more of propellant, but with the possibility that any emplaced lunar infrastructure can be amortized across multiple missions and other lunar industries whereas propellant delivered from Earth would be fixed requirement for every mission. The ISRU plant, tankers, etc. are in effect reusable propellant - in this sense. As with the price points, economic efficiency and performance efficiency are highly dependent on the degree of autonomy of the ISRU capability. Any mass of crew, their cargo, spare parts, or repair and replacement activities reduces the opportunity for the ISRU capability to compete on economics or performance against propellant tonnage costs in the case of all the propellant from Earth or at some other point (LEO depots).

Lastly, the costs and the price points for propellant are consistent with the advances in costs that have already been estimated using commercial acquisition approaches in NASA. Were NASA to apply a COTS-like acquisition approach beyond the launch vehicles or spacecraft (crew or cargo) to date, to other space systems (ISRU, lunar landers, orbital depots, etc.), the process incentives and characteristics open the door to the potential affordability improvements as was discussed earlier. In either case, even with the uncertainties of a COTS-like approach, traditional alternatives (cost-plus, etc.) carry the potential for costs so many times higher as to close the door to any human Mars missions at all given NASA's budgetary constraints.

\section{Concluding Remarks}

In accordance with the ultimate goal for NASA of sending humans to the surface of Mars, an economical and sustainable approach to achieving this goal was discussed and several recommendations were made. The key elements for this approach included 1)leveraging international and commercial participation as much as possible; 2)

learning to "live off the land" and developing ISRU capabilities to take advantage of resources existent on other worlds; 3) showing value and economic benefit to important stakeholders and the public to maintain financial 
support and achieve sustainability; 4) using Cis-Lunar space as a nearby testbed for maturing and demonstrating capabilities that will be essential for future Mars transportation systems and 5) creating a thriving Cis-Lunar economy to support and sustain future human missions to Mars.

To address all these elements, the authors recommended to initiate an LCOTS program using a COTS-like model that was established in 2005-06. The proposed LCOTS program as described, plans for NASA to enter into commercial space partnerships via Space Act Agreements with industry to demonstrate cost-effective, cis-lunar commercial services. These services may range from lunar transportation, to lunar ISRU operations, and cis-lunar propellant depots. Similar to the original COTS program, the goals of the LCOTS program will be to: 1) reduce development and operational costs by sharing costs with industry; 2) create new markets in cis-lunar space to further reduce operational costs; and 3) enable NASA to develop an affordable and economical exploration architecture.

A phased-development approach was also recommended to allow for incremental development and demonstration of capabilities. This approach was presented as a lower-risk approach since it allows for several offramps throughout the program and time for pause between phases to make sure its milestones and objectives are being met before proceeding to the next phase. The goals and objectives for each phase were presented along with options to initiate each phase.

Finally, an economic, business case was presented to assess viability of a business plan to produce lunar-derived propellant for a Mars transportation system. It was shown that for a propellant price point of $\$ 10 \mathrm{~K} / \mathrm{kg}$, it was feasible to close the business case for operating costs in the range of $\$ 6500 / \mathrm{kg}$ using autonomous operations with limited human tending. These estimates were based on a recent report from the ELA study ${ }^{11}$ to build and operate ISRU facilities on the surface of the Moon. This study resulted in estimated costs of \$40B over a 10-12 year period to establish a permanent commercial lunar base that can create and deliver up to $200 \mathrm{mt}$ of propellant per year to cislunar space. In addition, potential economic, performance and risk reduction benefits were described for a lunar ISRU plant that could benefit not only future human missions to Mars but to the emergence of cis-lunar industries as well.

Follow-on work was also recommended to continue evaluating the maturity of cis-lunar capabilities and technical and financial viability of emerging space companies before entering into commercial space partnerships for development and demonstration. Other key follow-on work included maturing the implementation options for each phase to meet program's schedule and budget constraints and continued work on the economic assessment of lunar-derived propellant for Mars missions including performing market studies to better predict the feasibility and likelihood of new cis-lunar industries.

In conclusion, it is recommended that a potential LCOTS program continue to be investigated to define a program plan that pursues a low-risk approach and yields the highest value and benefits towards reaching our ultimate goal of settling humans on Mars. As preliminary findings have indicated, a future LCOTS program has the great potential of enabling development of cost-effective, commercial capabilities and establishing a thriving cislunar economy which will lead the way to an economical and sustainable approach for future human missions to Mars.

\section{Acknowledgments}

The authors would like to acknowledge several members of the Commercial Space Group and Ames Space Portal who contributed to this paper including Dave Cheuvront, Mark Nall, Alan Wilhite, Lynn Harper, and Mark Newfield. Authors would also like to acknowledge the Ames management team for supporting this work including Eugene Tu, Gary Martin, Rose Grymes and Krishnakumar Kalmanje.

\section{References}

\footnotetext{
1 "National Space Policy of the United States of America," Office of the President of the United States, Washington D.C. URL: https://www.whitehouse.gov/sites/default/files/national_space_policy_6-28-10.pdf [cited 28 June 2010].

2 “NASA Strategic Plan 2014," NASA Headquarters, Washington D.C. URL: https://www.nasa.gov/sites/default/files/files/FY2014_NASA_SP 508c.pdf [cited 2014].

${ }^{3}$ Williams, G., Crusan, J., "Pioneering Space - the Evolvable Mars Campaign," NASA Headquarters, Washington, D.C. URL: https://www.nasa.gov/sites/default/files/files/20150408-NAC-Crusan-EMC-v7a.pdf [cited April 2015].

${ }^{4}$ Mars Architecture Steering Group, "Human Exploration of Mars Design Reference Architecture 5.0, Bret Drake, Editor, NASA/SP-2009-566, 2009.
} 
${ }^{5}$ National Research Council, "Pathways to Exploration: Rationales and Approaches for a US Program of Human Space Exploration,” The National Academies Press, Washington D.C., 2014.

${ }^{6}$ International Space Exploration Coordination Group, “The Global Exploration Roadmap,” August 2013.

${ }^{7}$ Price, H., Baker, J., Naderi, F., "A Scenario for a Human Mission to Mars Orbit in the 2030s, Thoughts Toward an Executable Program," Future In-Space Operations Telecom, May 2015. URL:

http://spirit.as.utexas.edu/ fiso/telecon.cgi [cited July 16, 2015]

8 Zapata, E., Wilhite, A., "Exploring NASA Human Spaceflight and Pioneering Scenarios", AIAA Joint Propulsion Conference, July 2015.

${ }^{9}$ Schrunk, D., Sharpe, B., Cooper, B., Thangavelu, M., "Lunar Resources," and "In-Situ Resource Utilization(ISRU)," The Moon, Resources, Future Development and Settlement, Second Edition, Praxis Publishing Ltd., Chichester, UK 2008.

${ }^{10}$ Crawford, I., "Lunar Resources: A Review", Progress of Physical Geography, 2014

${ }^{11}$ Miller, C., Wilhite, A., Cheuvront, D. (et al), "Economic Assessment and Systems Analysis of an Evolvable Lunar Architeture that Leverages Commercial Space Capabilities and Public-Private-Partnerships," NexGen Space LLC under a grant from NASA, 2015. URL: http://science.ksc.nasa.gov/shuttle/nexgen/rlvhp.htm [cited 22 July 2015].

${ }^{12}$ Sanders, "Lunar Polar ISRU as a Stepping Stone for Human Exploration," presented to Lunar Exploration Analysis Group (LEAG) Workshop, Oct 2013

${ }^{13}$ Spudis, P.D., Lavoie, A.R., "Using the resources of the Moon to create a permanent, cislunar space faring system,” AIAA 2011-7185, AIAA Space 2011 Conference \& Exposition, 27-29 Sep 2011, Long Beach, CA.

${ }^{14}$ Borowski, S., McCurdy, D., Packard, T., "7-Launch NTR Space Transportation System for NASA's Mars Design Reference Architecture (DRA) 5.0," $45^{\text {th }}$ AIAA/ASME/SAE/ASEE Joint Propulsion Conference, 2-5 August 2009, Denver, CO.

${ }^{15}$ Holladay, J., Jaap, J.P., Pinson, R.M., Creech, S.D. (et al), “Ares V Utilization in Support of a Human Mission to Mars,” NASA TM 2010-216450, NASA Marshall Space Center, Huntsville, AL, November 2010.

${ }^{16}$ Glidden, G., Wilhite, A., "Performance of Existing Launch Vehicle Stages for Earth Departure with Refuel from Orbital Propellant Depot," AIAA Space 2014 Conference and Exhibition, August 2014.

${ }^{17}$ Zegler, F., Kutter, B., "Evolving to a Depot-Based Space Transportation Architecture, “ AIAA Space 2010 Conference, AIAA-2010-7160, 2011.

${ }^{18}$ Spudis, P.D., Bussey, D.B.J., Baloga, S.M. (et al), "Evidence for water ice on the Moon: Results for anomalous polar craters from the LRO Mini-RF imaging radar," Journal of Geophysical Research: Planets, Vol. 118, 20162029, 2013.

${ }^{19}$ Williams, D. R., "Ice on the Moon - A Summary of Clementine and Lunar Prospector Results," NASA, URL: http://nssdc.gsfc.nasa.gov/planetary/ice/ice_moon.html [cited 12 May 2014].

${ }^{20}$ Colaprete, A., Schultz, P., Heldmann, J. (et al), "Detection of Water in the LCROSS Ejecta Plume," SCIENCE Journal, Vol. 30, No. 6003, 2010, pp. 463-468.

${ }^{21}$ McGovern J., Bussey D., Greenhagen B., Paige D., Cahill JTS and Spudis PD (2013) Mapping and characterization of non-polar permanent shadows on the lunar surface. Icarus 223: 566-581.

${ }^{22}$ Andrews, D., Colaprete, A., Quinn, J. (et al), "Introducing the Resource Prospector (RP) Mission”, AIAA Space 2014 Conference \& Exhibition, Sep 2014.

${ }^{23}$ Hecht, M.H. and Hoffman, J.A., "The Mars Oxygen ISRU Experiment on the Mars 2020 Rover," $46^{\text {th }}$ Lunar and Planetary Science Conference, 2015.

${ }^{24}$ Larson, W.E., Picard, M., Sanders, G.B., "RESOLVE: Ground Truth for Polar Volatiles as a Resource,” $62^{\text {nd }}$ International Astronautical Congress, Cape Town, South Africa, Oct 2011. 
${ }^{25}$ NASA, "Commercial Market Assessment for Crew and Cargo Systems Pursuant to Section 403 of the NASA Authorization Act of 2010 (P.L. 111-267)", April 27, 2011. URL:

http://www.nasa.gov/sites/default/files/files/Section403(b)CommercialMarketAssessmentReportFinal.pdf

${ }^{26}$ NASA, "Falcon 9 Launch Vehicle NAFCOM Cost Estimates", August 2011, NASA Assoc. Deputy Administrator for Policy, http://www.nasa.gov/pdf/586023main_8-3-11_NAFCOM.pdf

${ }^{27}$ Smrekar, S., et al., Deep Space 2: The Mars Microprobe Mission, J. Geophys. Res., 104, No. E11, 27013-27030, Nov. 1999

28 "Astrobotics Payload User Guide," Version 3.0, Pittsburgh, PA. URL: https://www.astrobotic.com/ [cited 2015]

${ }^{29}$ Blair, B., Lamassoure, E., Oderman, M., "Space Resource Economic Analysis Toolkit: The Case for Commercial Lunar Ice Mining, Final Report to the NASA Exploration Team" 2002.

${ }^{30}$ Griffin, M., "NASA and the Business of Space," American Astronautical Society, 2005. URL: https://www.nasa.gov/pdf/138033main_griffin_aas1.pdf [cited 13 July 2015].

${ }^{31}$ Zapata, E., "The LCC Model (Cost Data Sheets)" NASA, URL:

http://science.ksc.nasa.gov/shuttle/nexgen/Models/LCC.htm [cited July 16, 2015]

${ }^{32}$ Stanley, D., Wilhite, A., Zapata, E., "Propellant Depot Requirement Study, Status Report," NASA. URL: http://images.spaceref.com/news/2011/21.jul2011.vxs.pdf [cited 13 July 2015].

${ }^{33}$ Xingmeng, X., Green, J., Han, D., et al, “A Low-Cost, Large-Scale, Near-Term, Economically Sustainable Lunar Colony," University of Southern California, NASA 2015 RAASC-AL Competition, Cocoa Beach, FL, Revision A, June 23, 2015.

${ }^{34}$ De Weck, O., Hoffman, J., Ishimatsu, T., Schreiner, S., Ho, K., Owens, A., "Systems Impact of ISRU on a Human Mars Campiagn,” 2015. 